Article

\title{
Iminopyridine-Based Cobalt(II) and Nickel(II) Complexes: Synthesis, Characterization, and Their Catalytic Behaviors for 1,3-Butadiene Polymerization
}

\author{
Quanquan Dai ${ }^{\dagger}$, Xiangyu Jia ${ }^{\dagger}$, Feng Yang, Chenxi Bai ${ }^{*}$, Yanming $\mathrm{Hu}{ }^{*}$ and Xuequan Zhang \\ Received: 22 December 2015; Accepted: 7 January 2016; Published: 12 January 2016 \\ Academic Editor: Changle Chen \\ Key Laboratory of Synthetic Rubber, Changchun Institute of Applied Chemistry, Chinese Academy of Sciences, \\ Changchun 130022, China; qqdai@ciac.ac.cn (Q.D.); xiangyu_j@163.com (X.J.); yf18231@ciac.ac.cn (F.Y); \\ xqzhang@ciac.ac.cn (X.Z.) \\ * Correspondence: baicx@ciac.ac.cn (C.B.); ymhu@ciac.ac.cn (Y.H.); \\ Tel.: +86-431-85262304 (C.B.); +86-431-85262305 (Y.H.) \\ + These authors contributed equally to this work.
}

\begin{abstract}
A series of iminopyridine ligated $\mathrm{Co}(\mathrm{II})(\mathbf{1} \mathbf{a}-\mathbf{7 a})$ and $\mathrm{Ni}(\mathrm{II})(\mathbf{1} \mathbf{b}-\mathbf{7} \mathbf{b})$ complexes were synthesized. The structures of complexes $\mathbf{3 a}, \mathbf{4 a}, \mathbf{5 a}, \mathbf{7} \mathbf{a}, \mathbf{5} \mathbf{b}$, and $\mathbf{6 b}$ were determined by X-ray crystallographic analyses. Complex $3 \mathbf{a}$ formed a chloro-bridged dimer, whereas $\mathbf{4 a}, \mathbf{5 a}$, and $\mathbf{7 a}$, having a substituent $\left(4 \mathbf{a}, 5 \mathbf{a}: \mathrm{CH}_{3} ; \mathbf{7 a}\right.$ : $\left.\mathrm{Br}\right)$ at the 6-position of pyridine, producing the solid structures with a single ligand coordinated to the central metal. The nickel atom in complex $5 \mathbf{b}$ features distorted trigonal-bipyramidal geometry with one THF molecule ligating to the metal center. All the complexes activated by ethylaluminum sesquichloride (EASC) were evaluated in 1,3-butadiene polymerization. The catalytic activity and selectivity were significantly influenced by the ligand structure and central metal. Comparing with the nickel complexes, the cobalt complexes exhibited higher catalytic activity and cis-1,4-selectivity. For both the cobalt and nickel complexes, the aldimine-based complexes showed higher catalyst activity than their ketimine counterparts.
\end{abstract}

Keywords: iminopyridine; cobalt; nickel; 1,3-butadiene; polybutadiene

\section{Introduction}

Conjugated diene polymerization is a long-standing research subject of considerable interest from the viewpoints of fundamental and practical perspectives. 1,3-butadiene (BD) polymerization is capable of producing polybutadienes (PBDs) with different isomeric forms such as cis-1,4, trans-1,4, 1,2-syndiotactic, 1,2-isotactic, and 1,2-atactic, and each exhibits different properties and consequently find diverse applications [1]. Due to the elastomeric character, cis-1,4-polybutadiene is one of the most important raw materials in rubber industry. Industrially, Ziegler-Natta catalysts based on $\mathrm{Ti}, \mathrm{Nd}, \mathrm{Co}$, $\mathrm{Ni}$, and other transition metals are used in the production of cis-1,4-PBDs [2-4].

Cobalt-based catalysts attract special interests as the microstructure of the product, including cis-1,4-PBD and syndiotactic 1,2-PBD, depending on the structure of the ligand coordinated to central metal. As a result, a variety of cobalt complexes have been designed and exploited for cis-1,4 and 1,2-selected polymerization, such as the cobalt halides and carboxylates, or the combination of alkylphosphines and pyridyl adducts as electron donors when activated by methylaluminoxane (MAO) [5,6]. Because of their ill-defined nature, these multi-site catalysts often produced polymers with broad molecular weight distribution, resulting in poor mechanical properties. In order to gain better control over molecular weight, molecular weight distribution, and, more importantly, stereoselectivity of the polymerization, academic and industrial research has focused 
on well-defined organometallic single-site catalysts. For example, four-coordinated (salen)cobalt(II) $\left([\mathrm{ONNO}]^{2-}\right)[7]$ and bis(salicylaldiminate)cobalt(II) [8], three-coordinated bis(imino)pyridine cobalt (II) [9], bis(benzimidazolyl)amine cobalt (II) [10], bis(benzimidazolyl) pyridine [11,12] cobalt(II), bis(thiazolinyl)pyridine cobalt(II) [13], 2-arylimino-6-(alcohol)pyridine/2-arylamino-6-(alcohol) pyridine cobalt(II) [14], and 3-aryliminomethyl-2-hydroxybenzaldehyde cobalt(II) [15] exhibited high activity and high cis-1,4 selectivity in 1,3-butadiene polymerization in combination with MAO or EASC. The nickel-based catalysts are also of particular interest in the production of high cis-1,4-PBD. For example, (salen)nickel(II) ([ONNO $]^{2-}$ ) [16], nickel-tropolonoide, and nickel-1,3-propanedionate $\left([\mathrm{OO}]^{-}\right)$[17] afforded polymers with high cis-1,4 content, whereas nickel dihalide complexes bearing neutral $\alpha$-diimine ligands ([NN]) $[16,18]$ or bis(imino)pyridine ligands ([NNN]) $[17,19]$ were less active for the polymerization of 1,3-butadiene. Li et al. found that 2-arylimino-6-(alcohol)pyridine/2-arylamino-6-(alcohol)pyridine nickel (II) [14] produced the polymers with low molecular weight $\left(M_{n}=6000-9000\right)$ in high yields and the molecular weight distributions were somewhat broad $\left(M_{\mathrm{w}} / M_{\mathrm{n}}=3.18-4.25\right)$, while the ligand environments did not influence the stereoregularity of the resulting polymers.

We have been interested in the synthesis of transition metal complexes to promote 1,3-butadiene polymerization over the past decades. For example, cis-1,4 selective polymerization of 1,3-butadiene was achieved by bis(imino)pyridine cobalt(II) complexes/MAO catalyst [9], and the incorporation of electron-withdrawing groups could enhance activity and selectivity simultaneously. Moreover, other catalyst systems usually focused on the steric and electronic effects of the substitutions at the iminoaryl rings on the catalytic activity and properties of resulting polymers. Even though some of these complexes based on the iminopyridine ligands have been reported for ethylene polymerization and oligomerization [20-24], iminopyridine cobalt(II) and nickel(II) complexes for 1,3-butadiene polymerization have not been investigated yet. In the present study, iminopyridine cobalt(II) and nickel(II) complexes were synthesized, and the influences of the substituents at the 6-position of the pyridine ring and in the imino bridge on catalytic activity and selectivity in 1,3-butadiene polymerization were investigated.

\section{Materials and Methods}

\subsection{General Considerations and Materials}

All the manipulations were carried out in a nitrogen atmosphere by using standard Schlenk techniques. 1,3-Butadiene (Jinzhou Petrochemical Company, Jinzhou, China) was purified by passing through two columns containing potassium hydroxide and active alumina. Hexane (Beijing Reagents Factory, Beijing, China) was refluxed over sodium benzophenone ketyl until the solution turned blue, and then distilled before use. Ethylaluminum sesquichloride (EASC) was purchased from Acros Chemicals (Geel, Belgium). $\mathrm{CoCl}_{2}, \mathrm{NiBr}_{2}$ and all the anilines were obtained from Alfa Aesar (Ward Hill, UK). Other chemicals were commercially available and used without further purification.

FTIR spectra were performed on a BRUKE Vertex-70 FTIR spectrometer (Bruker Optics, Ettlingen, Germany). Elemental analysis was performed using an elemental Vario EL spectrophotometer (Elementar, Hanau, Germany). The molecular weights $\left(M_{\mathrm{w}}\right.$ and $\left.M_{\mathrm{n}}\right)$ and molecular weight distributions $\left(M_{\mathrm{w}} / M_{\mathrm{n}}\right)$ of polymers were measured at $30^{\circ} \mathrm{C}$ by gel permeation chromatography (GPC) equipped with a Waters 515HPLC pump, a series of four columns (HMW7THF, HMW6ETHF(two), HMW2THF) and a Waters 2414 refractive index detector (Waters, Massachusetts, UK). Tetrahydrofuran was used as an eluent at a flow rate of $1.0 \mathrm{~mL} / \mathrm{min}$.

X-ray crystallography measurements. Single crystals of $\mathbf{3 a}, \mathbf{4 a}, \mathbf{5 a}, \mathbf{7 a}, \mathbf{5 b}$, and $\mathbf{6 b}$ suitable for $X$-ray diffraction were obtained by a slow diffusion of diethyl ether into their THF solution. Data collections were performed at $-85^{\circ} \mathrm{C}$ on a BRUKE SMART APEX diffractometer (Bruker, Bremen, Germany) with a CCD area detector, using graphite monochromated Mo K radiation $(\lambda=0.71073 \AA)$. The determination of crystal class and unit cell parameters was carried out by the SMART program 
package. The raw frame data were processed using SAINT and SADABS to yield the reflection data file. The structures were solved by using SHELXTL program. Refinement was performed on $\mathrm{F}^{2}$ anisotropically for all non-hydrogen atoms by the full-matrix least-squares method. The hydrogen atoms were placed at the calculated positions and were included in the structure calculation without further refinement of the parameters.

\subsection{Procedure for 1,3-Butadiene Polymerization}

A typical procedure for the polymerization is as follows: a toluene solution of 1,3-butadiene $\left(1.0 \mathrm{~g}, 1.85 \times 10^{-2} \mathrm{~mol}\right)$ was added to a moisture free ampoule preloaded with complex $1 \mathrm{a}(7.3 \mathrm{mg}$, $\left.1.85 \times 10^{-5} \mathrm{~mol}\right)$, and EASC $\left(1.156 \times 10^{-3} \mathrm{~mol} / \mathrm{mL}, 0.4 \mathrm{~mL}\right)$ was then injected to initiate the polymerization at $20^{\circ} \mathrm{C}$. After $15 \mathrm{~min}$, methanol was added to the system to quench the polymerization. The mixture was poured into a large quantity of methanol containing 2,6-di-tertbutyl-4-methylphenol $(1.0 \mathrm{wt} \%)$ as a stabilizer. Via filtering and drying under vacuum at $40^{\circ} \mathrm{C}$, polybutadiene resulted at a constant weight $(0.569 \mathrm{~g}, 56.9 \%)$.

\subsection{Synthesis of Ligand (L1-L7)}

\subsubsection{2,6-Bis(1-methylethyl)-N-(2-pyridinylmethylene)phenylamine (L1)}

Ligand L1 was prepared according to the reported method [21]. Pyridine-2-carbaldehyde (2.0 g, $0.0187 \mathrm{~mol}$ ) was dissolved in $30 \mathrm{~mL}$ of methanol, then 2,6-di(1-methylethyl)phenylamine ( $3.55 \mathrm{~g}$, $0.02 \mathrm{~mol}$ ), and a few drops of formic acid were subsequently added. This mixture was refluxed for $12 \mathrm{~h}$. The solvent was evaporated in vacuo. The remaining crude product was dissolved in $n$-pentane, and the solution was dried with $\mathrm{Na}_{2} \mathrm{SO}_{4}$. After filtration, the compound $\mathbf{L 1}$ was crystallized from n-pentane at $-25{ }^{\circ} \mathrm{C}$, producing yellow crystals. Yield: $3.75 \mathrm{~g}(75.4 \%) .{ }^{1} \mathrm{H} \mathrm{NMR}\left(400 \mathrm{MHz}, \mathrm{CDCl}_{3}, \delta\right.$, ppm): 1.17 (d, $\left.12 \mathrm{H}, \mathrm{CHMe}_{2}\right), 2.97\left(\mathrm{~m}, 2 \mathrm{H}, \mathrm{CHMe}_{2}\right), 7.11-7.28\left(\mathrm{~m}, 3 \mathrm{H}, \mathrm{H}_{\text {phenyl }}\right), 7.41\left(\mathrm{t}, 1 \mathrm{H}, \mathrm{H}_{\text {pyridine, } 5}\right)$, $7.85\left(\mathrm{t}, 1 \mathrm{H}, \mathrm{H}_{\text {pyridine }, 4}\right), 8.26\left(\mathrm{~d}, 1 \mathrm{H}, \mathrm{H}_{\text {pyridine, } 3}\right), 8.31(\mathrm{~s}, 1 \mathrm{H}, \mathrm{CH}=\mathrm{N}), 8.72\left(\mathrm{~d}, 1 \mathrm{H}, \mathrm{H}_{\text {pyridine, } 6}\right) .{ }^{13} \mathrm{C} \mathrm{NMR}$ (100 MHz, $\left.\mathrm{CDCl}_{3}, \delta, \mathrm{ppm}\right): 162.80(\mathrm{CH}=\mathrm{N}), 149.51,148.22,137.05,136.54,125.12,124.30,122.88,122.57$, 121.14, $27.77\left(\mathrm{CHMe}_{2}\right), 23.27\left(\mathrm{CHMe}_{2}\right)$. IR $\left(\mathrm{KBr}, \mathrm{cm}^{-1}\right): 2959,1633,1586,1470,1385,808,779,754$. Anal. Calcd. For $\mathrm{C}_{18} \mathrm{H}_{22} \mathrm{~N}_{2}$ : C, 81.16; H, 8.32; N, 10.52. Found: C, 81.59; H, 7.96; N, 10.45.

\subsubsection{2,6-Bis(1-methylethyl)- $N$-[1-(2-pyridinyl)-ethylidene]phenylamine (L2)}

The procedure as above in (4.2.1) by using 2-acetyl-pyridine $(1.0 \mathrm{~g}, 0.0083 \mathrm{~mol})$ and 2,6-di(1-methylethyl)phenylamine ( $1.77 \mathrm{~g}, 0.01 \mathrm{~mol})$ produced $\mathbf{L} 2$ as yellow compound crystallized from methanol at $-25^{\circ} \mathrm{C}$ in $82.2 \%$ yield. ${ }^{1} \mathrm{H}$ NMR $\left(400 \mathrm{MHz}, \mathrm{CDCl}_{3}, \delta, \mathrm{ppm}\right): 1.16\left(\mathrm{~d}, 12 \mathrm{H}, \mathrm{CHMe}_{2}\right)$, $2.21\left(\mathrm{~s}, 3 \mathrm{H}, \mathrm{C}\left(\mathrm{CH}_{3}\right)=\mathrm{N}\right), 2.75\left(\mathrm{~m}, 2 \mathrm{H}, \mathrm{CHMe}_{2}\right), 7.07-7.15\left(\mathrm{~m}, 3 \mathrm{H}, \mathrm{H}_{\text {phenyl }}\right), 7.39\left(\mathrm{t}, 1 \mathrm{H}, \mathrm{H}_{\text {pyridine, } 5}\right)$, $7.79\left(\mathrm{t}, 1 \mathrm{H}, \mathrm{H}_{\text {pyridine }, 4}\right), 8.35\left(\mathrm{t}, 1 \mathrm{H}, \mathrm{H}_{\text {pyridine, } 3}\right) 8.69\left(\mathrm{t}, 1 \mathrm{H}, \mathrm{H}_{\text {pyridine, }}\right) .{ }^{13} \mathrm{C} \mathrm{NMR}\left(100 \mathrm{MHz}, \mathrm{CDCl}_{3}\right.$, $\delta, \mathrm{ppm}): 166.77(\mathrm{CMe}=\mathrm{N}), 156.29,148.39,146.25,136.25,135.58,124.57,123.40,122.80,121.11,28.06$ $\left(\mathrm{CHMe}_{2}\right), 23.03\left(\mathrm{CHMe}_{2}\right), 22.69\left(\mathrm{CHMe}_{2}\right), 17.11(\mathrm{C}(\mathrm{CH} 3)=\mathrm{N}) . \mathrm{IR}\left(\mathrm{KBr}, \mathrm{cm}^{-1}\right): 2958,1637,1585,1566$, 1363, 933, 824, 775, 743. Anal. Calcd. For $\mathrm{C}_{19} \mathrm{H}_{24} \mathrm{~N}_{2}$ : C, 81.38; H, 8.63; N, 9.99. Found: C, 81.78; $\mathrm{H}, 8.24$; N, 9.98 .

\subsubsection{2,6-Bis(1-methylethyl)- $N$-(phenyl-2-pyridinylmethylene)phenylamine (L3)}

Phenyl-2-pyridinylmethanone $(2.0 \mathrm{~g}, 0.01 \mathrm{~mol})$ was dissolved in $30 \mathrm{~mL}$ of methanol, then 2,6-di(1-methylethyl)phenylamine $(1.95 \mathrm{~g}, 0.011 \mathrm{~mol})$ and a few drops of concentrated $\mathrm{H}_{2} \mathrm{SO}_{4}$ were added. This mixture was refluxed for $12 \mathrm{~h}$. The solvent was evaporated in vacuo. The yellow raw product was dissolved in $50 \mathrm{~mL}$ of dichloromethane, filtered, washed with $50 \mathrm{~mL}$ of water and dried with $\mathrm{Na}_{2} \mathrm{SO}_{4}$. After filtration and solvent evaporation, the yellow residue was purified by crystallization from methanol at $-25^{\circ} \mathrm{C}$, affording yellow, needle-like crystals in $46.7 \%$ yield. ${ }^{1} \mathrm{H} N \mathrm{NR}$ (400 MHz, $\left.\mathrm{CDCl}_{3}, \delta, \mathrm{ppm}\right): 0.89$ (d, $\left.3 \mathrm{H}, \mathrm{CHMe}_{2}\right), 0.96\left(\mathrm{~d}, 3 \mathrm{H}, \mathrm{CHMe}_{2}\right), 1.13\left(\mathrm{~d}, 6 \mathrm{H}, \mathrm{CHMe}_{2}\right), 2.86(\mathrm{~m}$, $\left.1 \mathrm{H}, \mathrm{CHMe}_{2}\right), 2.96\left(\mathrm{~m}, 1 \mathrm{H}, \mathrm{CHMe}_{2}\right), 6.96-7.21\left(\mathrm{~m}, 6 \mathrm{H}, \mathrm{H}_{\text {arom }}\right), 7.44\left(\mathrm{~m}, \mathrm{H}_{\text {arom }}\right), 7.81\left(\mathrm{~m}, \mathrm{H}_{\text {arom }}\right)$, 
$8.23\left(\mathrm{~d}, \mathrm{H}_{\text {arom }}\right), 8.61\left(\mathrm{~d}, \mathrm{H}_{\text {arom }}\right) .{ }^{13} \mathrm{C}$ NMR $\left(100 \mathrm{MHz}, \mathrm{CDCl}_{3}, \delta, \mathrm{ppm}\right): 165.26(\mathrm{C}($ Phenyl $)=\mathrm{N}), 164.04$, $157.52,154.25,148.91,148.59,145.91,138.17,136.36,135.31,134.69,130.36,129.39,129.06,128.03,127.33$, 123.46, 123.09, 122.41, $28.15\left(\mathrm{CHMe}_{2}\right), 23.74\left(\mathrm{CHMe}_{2}\right), 23.64\left(\mathrm{CHMe}_{2}\right), 21.81\left(\mathrm{CHMe}_{2}\right), 21.71\left(\mathrm{CHMe}_{2}\right)$. IR $\left(\mathrm{KBr}, \mathrm{cm}^{-1}\right): 2955,1616,1584,1380,831,799$, 774. Anal. Calcd. For $\mathrm{C}_{24} \mathrm{H}_{26} \mathrm{~N}_{2}$ : C, 84.17; H, 7.65; N, 8.18. Found: $\mathrm{C}, 84.65 ; \mathrm{H}, 8.02 ; \mathrm{N}, 7.33$.

\subsubsection{2,6-Bis(1-methylethyl)- $N$-[(6-methyl-2-pyridinyl)-methylene]phenylamine (L4)}

The procedure as above in (4.2.1) by using 6-methyl-pyridine-2-carbaldehyde (1.0 g, $0.0083 \mathrm{~mol})$ and 2,6-di(1-methylethyl)phenylamine $(1.77 \mathrm{~g}, 0.01 \mathrm{~mol})$ produced $\mathbf{L} 4$ as yellow compound crystallized from methanol at $-25{ }^{\circ} \mathrm{C}$ in $92.6 \%$ yield. ${ }^{1} \mathrm{H} \mathrm{NMR}\left(400 \mathrm{MHz}, \mathrm{CDCl}_{3}, \delta, \mathrm{ppm}\right): 1.16\left(\mathrm{~d}, 12 \mathrm{H}, \mathrm{CHMe}_{2}\right)$, $2.64\left(\mathrm{~s}, 3 \mathrm{H}, \mathrm{H}_{\text {pyridine,6-Me }}\right), 2.97$ (m, $\left.2 \mathrm{H}, \mathrm{CHMe}_{2}\right)$, 7.09-7.28 (m, $\left.3 \mathrm{H}, \mathrm{H}_{\text {phenyl }}\right), 7.28\left(\mathrm{~d}, 1 \mathrm{H}, \mathrm{H}_{\text {pyridine, },}\right)$, $7.72\left(\mathrm{t}, 1 \mathrm{H}, \mathrm{H}_{\text {pyridine }, 4}\right), 8.07$ (d, $\left.1 \mathrm{H}, \mathrm{H}_{\text {pyridine, } 3)}\right), 8.27$ (s, $\left.1 \mathrm{H}, \mathrm{CH}=\mathrm{N}\right) .{ }^{13} \mathrm{C} \mathrm{NMR}\left(100 \mathrm{MHz}, \mathrm{CDCl}_{3}\right.$, $\delta, \mathrm{ppm}): 163.06(\mathrm{CH}=\mathrm{N}), 158.28,153.69,148.33,136.98,136.67,124.78,124.14,122.78,118.21,27.72$ ( $\left.\mathrm{CHMe}_{2}\right), 24.18$ ( $\left.\mathrm{C}_{\text {pyridine, 6-Me }}\right), 23.23$ ( $\left.\mathrm{CHMe}_{2}\right)$. IR $\left(\mathrm{KBr}, \mathrm{cm}^{-1}\right): 2959,1642,1588,1456,1318,856,795$, 748. Anal. Calcd. For $\mathrm{C}_{19} \mathrm{H}_{24} \mathrm{~N}_{2}$ : C, 81.38; H, 8.63; N, 9.99. Found: $\mathrm{C}, 81.26 ; \mathrm{H}, 8.85 ; \mathrm{N}, 9.89$.

\subsubsection{2,6-Bis(1-methylethyl)-N-[1-(6-methyl-2-pyridinyl)-ethylidene]phenylamine (L5)}

The procedure as above in (4.2.1) by using 6-methyl-2-acetylpyridine $(2.0 \mathrm{~g}, 0.0148 \mathrm{~mol})$ and 2,6-di(1-methylethyl)phenylamine ( $2.66 \mathrm{~g}, 0.015 \mathrm{~mol})$ produced $\mathbf{L} 5$ as yellow compound crystallized from methanol at $-25^{\circ} \mathrm{C}$ in $89.2 \%$ yield. ${ }^{1} \mathrm{H} \mathrm{NMR}\left(400 \mathrm{MHz}, \mathrm{CDCl}_{3}, \delta, \mathrm{ppm}\right): 1.13\left(\mathrm{~d}, 12 \mathrm{H}, \mathrm{CHMe}_{2}\right)$, $2.20\left(\mathrm{~s}, 3 \mathrm{H}, \mathrm{C}\left(\mathrm{CH}_{3}\right)=\mathrm{N}\right), 2.62\left(\mathrm{~s}, 3 \mathrm{H}, \mathrm{H}_{\text {pyridine, 6-Me }}\right), 2.75(\mathrm{~m}, 2 \mathrm{H}, \mathrm{CHMe} 2), 7.06-7.24\left(\mathrm{~m}, 3 \mathrm{H}, \mathrm{H}_{\text {phenyl }}\right)$, $7.24\left(\mathrm{~d}, 1 \mathrm{H}, \mathrm{H}_{\text {pyridine }, 5}\right), 7.68\left(\mathrm{t}, 1 \mathrm{H}, \mathrm{H}_{\text {pyridine }, 4}\right), 8.14\left(\mathrm{~d}, 1 \mathrm{H}, \mathrm{H}_{\text {pyridine }, 3}\right) .{ }^{13} \mathrm{C} \mathrm{NMR}\left(100 \mathrm{MHz}, \mathrm{CDCl}_{3}\right.$, $\delta$, ppm): $167.18(\mathrm{CH}=\mathrm{N}), 157.16,155.77,146.47,136.35,135.63,124.00,123.27,122.77,118.04,28.04$

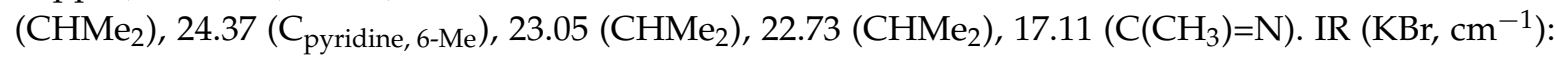
$2961,1643,1586,1458,1192,892,763,742$. Anal. Calcd. For $\mathrm{C}_{20} \mathrm{H}_{26} \mathrm{~N}_{2}: \mathrm{C}, 81.59 ; \mathrm{H}, 8.90 ; \mathrm{N}, 9.51$. Found: C, $81.83 ; \mathrm{H}, 9.33 ; \mathrm{N}, 8.84$.

\subsubsection{2,6-Bis(1-methylethyl)- $\mathrm{N}$-[(6-bromo-2-pyridinyl)-methylene]phenylamine (L6)}

6-Bromo-pyridine-2-carbaldehyde $(1.0 \mathrm{~g}, 0.005 \mathrm{~mol})$ was dissolved in $30 \mathrm{~mL}$ of methanol, then 2,6-di(1-methylethyl)phenylamine (1.06 g, $0.006 \mathrm{~mol})$, and a few drops of formic acid were subsequently added. This mixture was stirred at room temperature, a yellow precipitate was formed after $10 \mathrm{~min}$. The mixture was stirred for another $5 \mathrm{~h}$, then the precipitation was collected and washed twice with cold methanol, yielding the analytically pure compound L6 ( $1.64 \mathrm{~g}, 95.4 \%)$ as a yellow powder. ${ }^{1} \mathrm{H}$ NMR $\left(400 \mathrm{MHz}, \mathrm{CDCl}_{3}, \delta, \mathrm{ppm}\right): 1.15\left(\mathrm{~d}, 12 \mathrm{H}, \mathrm{CHMe}_{2}\right), 2.92\left(\mathrm{~m}, 2 \mathrm{H}, \mathrm{CHMe}_{2}\right), 7.11-7.18(\mathrm{~m}, 3 \mathrm{H}$, $\left.\mathrm{H}_{\text {phenyl }}\right)$, $7.60\left(\mathrm{~d}, 1 \mathrm{H}, \mathrm{H}_{\text {pyridine, }, 3}\right), 7.71\left(\mathrm{t}, 1 \mathrm{H}, \mathrm{H}_{\text {pyridine }, 4}\right), 8.24(\mathrm{~s}, 1 \mathrm{H}, \mathrm{CH}=\mathrm{N}), 8.26\left(\mathrm{~d}, 1 \mathrm{H}, \mathrm{H}_{\text {pyridine }, 5}\right)$. ${ }^{13} \mathrm{C}$ NMR $\left(100 \mathrm{MHz}, \mathrm{CDCl}_{3}, \delta, \mathrm{ppm}\right): 161.31(\mathrm{CH}=\mathrm{N}), 155.28,147.75,141.69,138.83,136.85,129.64$, 124.53, 122.90, 119.71, $27.78\left(\mathrm{CHMe}_{2}\right), 23.22\left(\mathrm{CHMe}_{2}\right) . \mathrm{IR}\left(\mathrm{KBr}, \mathrm{cm}^{-1}\right)$ : 2959, 1644, 1574, 1362, 1185, 809, 796, 763. Anal. Calcd. For $\mathrm{C}_{18} \mathrm{H}_{21} \mathrm{BrN}_{2}$ : C, 62.61; H, 6.13; N, 8.11. Found: $\mathrm{C}, 61.59 ; \mathrm{H}, 5.81 ; \mathrm{N}, 8.64$.

\subsubsection{2,6-Bis(1-methylethyl)- $\mathrm{N}$-[1-(6-bromo-2-pyridinyl)-ethylidene]phenylamine (L7)}

The procedure as above in (4.2.6) using 2-bromo-6-acetylpyridine $(1.0 \mathrm{~g}, 0.005 \mathrm{~mol})$ and 2,6-di(1-methylethyl)phenylamine $(0.975 \mathrm{~g}$, $0.0055 \mathrm{~mol})$ produced $\mathbf{L} 7$ as yellow compound in $74.6 \%$ yield. ${ }^{1} \mathrm{H}$ NMR (400 MHz, $\left.\mathrm{CDCl}_{3}, \delta, \mathrm{ppm}\right): 1.12-1.15\left(\mathrm{~m}, 12 \mathrm{H}, \mathrm{CHMe}_{2}\right), 2.18(\mathrm{~s}, 3 \mathrm{H}, \mathrm{C}(\mathrm{Me})=\mathrm{N})$, $2.69\left(\mathrm{~m}, 2 \mathrm{H}, \mathrm{CHMe}_{2}\right), 7.07-7.16\left(\mathrm{~m}, 3 \mathrm{H}, \mathrm{H}_{\text {phenyl }}\right), 7.56\left(\mathrm{~d}, 1 \mathrm{H}, \mathrm{H}_{\text {pyridine, } 3}\right), 7.65\left(\mathrm{t}, 1 \mathrm{H}, \mathrm{H}_{\text {pyridine }, 4}\right), 8.33$ $\left(\mathrm{d}, 1 \mathrm{H}, \mathrm{H}_{\text {pyridine, }, 5) .}{ }^{13} \mathrm{C}\right.$ NMR $\left(100 \mathrm{MHz}, \mathrm{CDCl}_{3}, \delta, \mathrm{ppm}\right): 165.72(\mathrm{C}(\mathrm{Me})=\mathrm{N}), 157.22,145.94,140.79$, 138.56, 135.44, 131.56, 128.98, 123.61, 122.83, 120.26, 119.83, $28.08\left(\mathrm{CHMe}_{2}\right), 23.01\left(\mathrm{CHMe}_{2}\right), 22.64$ $\left(\mathrm{CHMe}_{2}\right), 17.07(\mathrm{C}(\mathrm{Me})=\mathrm{N})$. IR $\left(\mathrm{KBr}, \mathrm{cm}^{-1}\right)$ : 2960, 1640, 1551, 1436, 1361, 1306, 834, 753, 734. Anal. Calcd. For $\mathrm{C}_{19} \mathrm{H}_{23} \mathrm{BrN}_{2}$ : C, 63.51; H, 6.45; N, 7.80. Found: C, 62.94; H, 6.62; N, 8.10. 


\subsection{Preparation of Cobalt and Nickel Complexes}

2.4.1. (2,6-Bis(1-methylethyl)-N-(2-pyridinylmethylene)phenylamine)cobalt(II) dichloride, 1a

A mixture of $\mathbf{L 1}(0.2 \mathrm{~g}, 0.00075 \mathrm{~mol})$ and anhydrous $\mathrm{CoCl}_{2}(0.1 \mathrm{~g}, 0.00075 \mathrm{~mol})$ were added to a flask containing $5 \mathrm{~mL}$ THF. The mixture was stirred at room temperature for $24 \mathrm{~h}$, a green suspension was formed. Diethyl ether was added and a suspension formed. The precipitate was collected by filtration and washed with $4 \times 5 \mathrm{~mL}$ heptane. The desired product $(0.21 \mathrm{~g}, 70.7 \%)$ was obtained after dried in vacuo at $40^{\circ} \mathrm{C}$. IR $\left(\mathrm{KBr}, \mathrm{cm}^{-1}\right)$ : 2968, 1625, 1593, 1465, 1383, 806, 775, 762. Anal. Calcd. For $\mathrm{C}_{18} \mathrm{H}_{22} \mathrm{Cl}_{2} \mathrm{CoN}_{2}$ : C, 54.56; H, 5.60; N, 7.07. Found: C, 53.86; H, 6.01; N, 7.26.

2.4.2. (2,6-Bis(1-methylethyl)- $N$-[1-(2-pyridinyl)-ethylidene]phenylamine)cobalt(II) dichloride, 2a

Procedure similar for $\mathbf{2 a}$ was adopted by using $\mathbf{L 2}$, and $\mathrm{CoCl}_{2}$ produced $\mathbf{2} \mathbf{a}$ as a green powder in $82.3 \%$ yield. IR $\left(\mathrm{KBr}, \mathrm{cm}^{-1}\right): 2968,1612,1591,1572,1373,939,846,783,753$. Anal. Calcd. For $\mathrm{C}_{19} \mathrm{H}_{24} \mathrm{Cl}_{2} \mathrm{CoN}_{2}$ : C, 55.63; $\mathrm{H}, 5.90 ; \mathrm{N}, 6.83$. Found: $\mathrm{C}, 56.03 ; \mathrm{H}, 6.17 ; \mathrm{N}, 6.65$.

\subsection{3. (2,6-Bis(1-methylethyl)-N-(phenyl-2-pyridinylmethylene)phenylamine) cobalt(II) dichloride, 3a}

Procedure similar for $\mathbf{3 a}$ was adopted by using $\mathbf{L 3}$, and $\mathrm{CoCl}_{2}$ produced $\mathbf{3 a}$ as a green powder in $52.8 \%$ yield. IR $\left(\mathrm{KBr}, \mathrm{cm}^{-1}\right): 2974,1609,1570,1384,800,778,765$. Anal. Calcd. For $\mathrm{C}_{24} \mathrm{H}_{26} \mathrm{Cl}_{2} \mathrm{CoN}_{2}$ : C, 61.03; H, 5.55; N, 5.93. Found: C, 61.24; H, 5.75; N, 6.02.

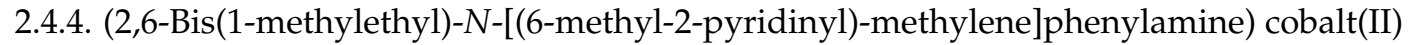
dichloride, $4 \mathbf{a}$

Procedure similar for $\mathbf{4 a}$ was adopted by using $\mathbf{L 4}$, and $\mathrm{CoCl}_{2}$ produced $\mathbf{4 a}$ as a green powder in $92.7 \%$ yield. IR $\left(\mathrm{KBr}, \mathrm{cm}^{-1}\right)$ : 2966, 1633, 1596, 1466, 1328, 866, 802, 742. Anal. Calcd. For $\mathrm{C}_{19} \mathrm{H}_{24} \mathrm{Cl}_{2} \mathrm{CoN}_{2}$ : C, 55.63; H, 5.90; N, 6.83. Found: C, 55.37; H, 6.22; N, 7.29.

2.4.5. (2,6-Bis(1-methylethyl)-N-[1-(6-methyl-2-pyridinyl)-ethylidene]phenylamine) cobalt(II) dichloride, $5 \mathbf{a}$

Procedure similar for $\mathbf{5 a}$ was adopted by using $\mathbf{L} \mathbf{5}$, and $\mathrm{CoCl}_{2}$ produced $\mathbf{5} \mathbf{a}$ as a green powder in $86.4 \%$ yield. IR $\left(\mathrm{KBr}, \mathrm{cm}^{-1}\right)$ : 2963, 1618, 1593, 1460, 1196, 888, 779, 740. Anal. Calcd. For $\mathrm{C}_{20} \mathrm{H}_{26} \mathrm{Cl}_{2} \mathrm{CoN}_{2}$ : C, 56.62; H, 6.18; N, 6.60. Found: C, 56.80; H, 6.03; N, 6.58.

2.4.6. (2,6-Bis(1-methylethyl)-N-[(6-bromo-2-pyridinyl)-methylene]phenylamine) cobalt(II) dichloride, $6 \mathbf{a}$

Procedure similar for $\mathbf{6 a}$ was adopted by using $\mathbf{L 6}$, and $\mathrm{CoCl}_{2}$ produced $\mathbf{6 a}$ as a green powder in $91.2 \%$ yield. IR $\left(\mathrm{KBr}, \mathrm{cm}^{-1}\right)$ : 2961, 1630, 1584, 1365, 1177, 813, 803, 765 . Anal. Calcd. For $\mathrm{C}_{18} \mathrm{H}_{21} \mathrm{BrCl}_{2} \mathrm{CoN}_{2}: \mathrm{C}, 45.50 ; \mathrm{H}, 4.46 ; \mathrm{N}, 5.90$. Found: $\mathrm{C}, 44.72 ; \mathrm{H}, 4.77 ; \mathrm{N}, 6.28$.

2.4.7. (2,6-Bis(1-methylethyl)- $\mathrm{N}$-[1-(6-bromo-2-pyridinyl)-ethylidene]phenylamine) cobalt(II) dichloride, $7 \mathbf{a}$

Procedure similar for $7 \mathbf{a}$ was adopted by using $\mathbf{L 7}$, and $\mathrm{CoCl}_{2}$ produced $7 \mathbf{a}$ as a green powder in $88.1 \%$ yield. IR $\left(\mathrm{KBr}, \mathrm{cm}^{-1}\right): 2966,1619,1555,1445,1367,1311,859,755,730$. Anal. Calcd. For $\mathrm{C}_{19} \mathrm{H}_{23} \mathrm{BrCl}_{2} \mathrm{CoN}_{2}$ : C, 46.65; $\mathrm{H}, 4.74 ; \mathrm{N}$, 5.73. Found: $\mathrm{C}, 47.09 ; \mathrm{H}, 4.52 ; \mathrm{N}, 6.27$.

\subsection{8. (2,6-Bis(1-methylethyl)- $N$-(2-pyridinylmethylene)phenylamine)nickel(II) dibromide, $\mathbf{1 b}$}

To a suspension of $\mathrm{NiBr}_{2}(\mathrm{dme})(0.11 \mathrm{~g}, 0.00036 \mathrm{~mol})$ in $5 \mathrm{~mL}, \mathrm{CH}_{2} \mathrm{Cl}_{2}$ was added into the solution of ligand $\mathbf{L 1}(0.1 \mathrm{~g}, 0.00036 \mathrm{~mol})$ in $5 \mathrm{~mL} \mathrm{CH}_{2} \mathrm{Cl}_{2}$. The orange-red reaction mixture was formed and stirred for $24 \mathrm{~h}$ at room temperature, and the precipitate was collected by filtration, washed with pentane $(2 \times 10 \mathrm{~mL})$, and dried under vacuum to produce complex $\mathbf{1} \mathbf{b}$ as an orange-red powder. Yield: 
$0.15 \mathrm{~g}(83.6 \%)$. IR (KBr, cm $\left.{ }^{-1}\right)$ : 2964, 1630, 1596, 1387, 802, 775, 760. Anal. Calcd. For $\mathrm{C}_{18} \mathrm{H}_{22} \mathrm{Br}_{2} \mathrm{~N}_{2} \mathrm{Ni}$ : C, 44.59; H, 4.57; N, 5.78. Found: C, 44.69; H, 4.77; N, 5.44.

2.4.9. (2,6-Bis(1-methylethyl)-N-[1-(2-pyridinyl)-ethylidene]phenylamine)nickel(II) dibromide, $2 \mathbf{b}$

Procedure similar for $\mathbf{2} \mathbf{b}$ was adopted by using $\mathbf{L} \mathbf{2}$, and $\mathrm{NiBr}_{2}(\mathrm{dme})$ produced $\mathbf{2} \mathbf{b}$ as a green powder in $91.7 \%$ yield. IR (KBr, $\left.\mathrm{cm}^{-1}\right): 2966,1612,1591,1571,1373,940,816,781,752$. Anal. Calcd. For $\mathrm{C}_{19} \mathrm{H}_{24} \mathrm{Br}_{2} \mathrm{~N}_{2} \mathrm{Ni}$ : C, 46.74; $\mathrm{H}, 4.85 ; \mathrm{N}$, 5.61. Found: C, 46.99; H, 4.81; N, 5.87.

2.4.10. (2,6-Bis(1-methylethyl)-N-(phenyl-2-pyridinylmethylene)phenylamine) nickel(II) dibromide, 3b

Procedure similar for $\mathbf{3} \mathbf{b}$ was adopted by using $\mathbf{L} \mathbf{3}$, and $\mathrm{NiBr}_{2}(\mathrm{dme})$ produced $\mathbf{3} \mathbf{b}$ as a green powder in $47.5 \%$ yield. IR $\left(\mathrm{KBr}, \mathrm{cm}^{-1}\right)$ : 2964, 1632, 1596, 1379, 801, 776, 741. Anal. Calcd. For $\mathrm{C}_{24} \mathrm{H}_{26} \mathrm{Br}_{2} \mathrm{~N}_{2} \mathrm{Ni}$ : C, 51.38; H, 4.67; N, 4.99. Found: C, 51.06; H, 4.37; N, 5.12

2.4.11. (2,6-Bis(1-methylethyl)- $N$-[(6-methyl-2-pyridinyl)-methylene]phenylamine) nickel(II) dibromide, $4 \mathbf{b}$

Procedure similar for $\mathbf{4 b}$ was adopted by using $\mathbf{L} \mathbf{4}$, and $\mathrm{NiBr}_{2}(\mathrm{dme})$ produced $\mathbf{4 b}$ as a green powder in $76.7 \%$ yield. IR (KBr, cm $\left.{ }^{-1}\right)$ : Ni: 2962, 1632, 1597, 1467, 1330, 867, 800, 741. Anal. Calcd. For $\mathrm{C}_{19} \mathrm{H}_{24} \mathrm{Br}_{2} \mathrm{~N}_{2} \mathrm{Ni}$ : C, 45.74; $\mathrm{H}, 4.85 ; \mathrm{N}$, 5.61. Found: C, 45.85; H, 5.09; N, 4.62

2.4.12. (2,6-Bis(1-methylethyl)-N-[1-(6-methyl-2-pyridinyl)-ethylidene]phenylamine) nickel(II) dibromide, $5 \mathbf{b}$

Procedure similar for $\mathbf{5 b}$ was adopted by using $\mathbf{L} 5$, and $\mathrm{NiBr}_{2}(\mathrm{dme})$ produced $\mathbf{5 b}$ as a green powder in $83.4 \%$ yield. IR $\left(\mathrm{KBr}, \mathrm{cm}^{-1}\right)$ : Ni: 2965, 1617, 1595, 1461, 1196, 890, 779, 738. Anal. Calcd. For $\mathrm{C}_{20} \mathrm{H}_{26} \mathrm{Br}_{2} \mathrm{~N}_{2} \mathrm{Ni}$ : C, 46.83; $\mathrm{H}, 5.11 ; \mathrm{N}$, 5.46. Found: $\mathrm{C}, 47.24 ; \mathrm{H}, 5.34 ; \mathrm{N}, 5.71$

2.4.13. (2,6-Bis(1-methylethyl)-N-[(6-bromo-2-pyridinyl)-methylene]phenylamine) nickel(II) dibromide, $6 \mathbf{b}$

Procedure similar for $\mathbf{6 b}$ was adopted using $\mathbf{L 6}$, and $\mathrm{NiBr}_{2}(\mathrm{dme})$ produced $\mathbf{6 b}$ as a green powder in $93.6 \%$ yield. IR $\left(\mathrm{KBr}, \mathrm{cm}^{-1}\right)$ : 2961, 1626, 1585, 1365, 1175, 810, 800, 763. Anal. Calcd. For $\mathrm{C}_{18} \mathrm{H}_{21} \mathrm{Br}_{3} \mathrm{~N}_{2} \mathrm{Ni}$ : C, 38.35; H, 3.75; N, 4.97. Found: C, 37.86; H, 3.94; N, 5.12.

2.4.14. (2,6-Bis(1-methylethyl)-N-[1-(6-bromo-2-pyridinyl)-ethylidene]phenylamine) nickel(II) dibromide, $7 \mathbf{b}$

Procedure similar for $\mathbf{7 b}$ was adopted by using $\mathbf{L 7}$, and $\mathrm{NiBr}_{2}(\mathrm{dme})$ produced $\mathbf{7 b}$ as a green powder in $85.1 \%$ yield. IR (KBr, cm $\left.{ }^{-1}\right): 2965,1613,1553,1440,1369,1315,828,764,733$. Anal. Calcd. For $\mathrm{C}_{19} \mathrm{H}_{23} \mathrm{Br}_{3} \mathrm{~N}_{2} \mathrm{Ni}$ : C, 39.49; $\mathrm{H}, 4.01 ; \mathrm{N}$, 4.85. Found: C, 40.10; H, 3.71; N, 4.99.

\section{Results and Discussions}

\subsection{Synthesis and Characterization of Iminopyridine Ligands (L1-L7) and Their Cobalt(II) and Nickel(II) Complexes}

The iminopyridine ligands L1, L2, L4-L7 were prepared by the condensation of 2-pyridinecarboxaldehydes or 2-pyridylketones with 2,6-di(1-methylethyl)phenylamine in the presence of a catalytic amount of formic acid in methanol (Scheme 1). Ligand $\mathbf{L 3}$ was synthesized with a few drops of concentrated $\mathrm{H}_{2} \mathrm{SO}_{4}$ as catalyst, and anhydrous $\mathrm{Na}_{2} \mathrm{SO}_{4}$ was used to remove the water formed during the reaction according to the literature [21]. All the ligands were identified by FT-IR, elemental analysis, and NMR spectra. Cobalt complexes 1a-7a were prepared by the reaction of anhydrous cobalt(II) chloride with the corresponding ligand in THF. All of the complexes were isolated as green air-stable powders in high yields. Nickel complexes $\mathbf{1} \mathbf{b}-\mathbf{7 b}$ were prepared by the reaction of the 
corresponding ligand with $\mathrm{NiBr}_{2}(\mathrm{dme})$ in $\mathrm{CH}_{2} \mathrm{Cl}_{2}$. The structures of these complexes were determined by FT-IR spectra and elemental analysis. The structures of complexes $3 \mathbf{a}, \mathbf{4 a}, \mathbf{5 a}, \mathbf{7 a}, \mathbf{5 b}$, and $\mathbf{6 b}$ were further characterized by X-ray crystallographic analysis.

\subsection{Crystal Structure of Complexes}

Single crystals of $\mathbf{3 a}, \mathbf{4 a}, \mathbf{5 a}, \mathbf{7 a}, \mathbf{5 b}$, and $\mathbf{6 b}$ suitable for X-ray diffraction analysis were obtained by crystallization from their THF or diethyl ether solutions. The crystal data together with the data collection and structure refinement parameters are presented in Table 1. Selected bond lengths and angles for cobalt complexes (3a, $\mathbf{4 a}, \mathbf{5 a}$ and $\mathbf{7 a}$ ) are given in Table 2. Selected bond lengths and angles for nickel complexes ( $5 \mathbf{b}$ and $\mathbf{6 b}$ ) are given in Table 3 .

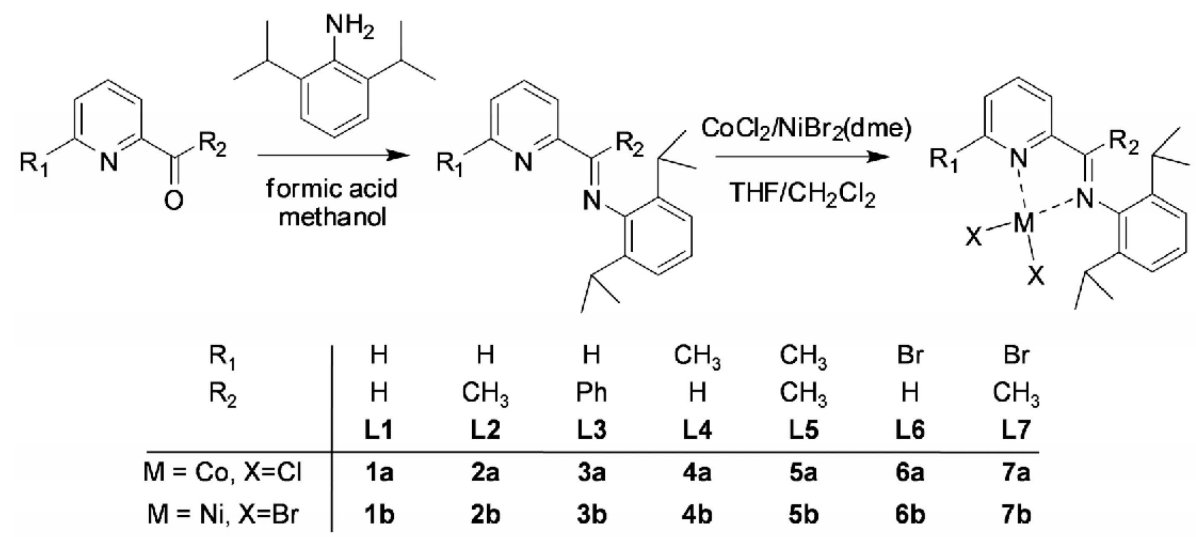

Scheme 1. Synthesis of cobalt complexes $\mathbf{1 a - 7 a}$ and nickel complexes $\mathbf{1 b}-\mathbf{7 b}$.

Table 1. Crystal data and data collection parameters of cobalt and nickel complexes.

\begin{tabular}{|c|c|c|c|c|c|c|}
\hline & $3 \mathrm{a} \cdot \mathrm{Et}_{2} \mathrm{O}$ & $4 a$ & $5 a$ & $7 a$ & $5 b \cdot$ THF & $6 b$ \\
\hline Formula & $\mathrm{C}_{52} \mathrm{H}_{62} \mathrm{Cl}_{4} \mathrm{Co}_{2} \mathrm{~N}_{4} \mathrm{O}$ & $\mathrm{C}_{19} \mathrm{H}_{24} \mathrm{Cl}_{2} \mathrm{CoN}_{2}$ & $\mathrm{C}_{20} \mathrm{H}_{26} \mathrm{Cl}_{2} \mathrm{CoN}_{2}$ & $\mathrm{C}_{19} \mathrm{H}_{23} \mathrm{BrCl}_{2} \mathrm{CoN}_{2}$ & $\mathrm{C}_{24} \mathrm{H}_{34} \mathrm{Br}_{2} \mathrm{~N}_{2} \mathrm{NiO}$ & $\mathrm{C}_{36} \mathrm{H}_{42} \mathrm{Br}_{6} \mathrm{~N}_{4} \mathrm{Ni}_{2}$ \\
\hline Molecular weight & $1,018.72$ & 410.23 & 424.26 & 489.13 & 585.06 & $1,127.62$ \\
\hline Wavelength $(\AA)$ & 0.71073 & 0.71073 & 0.71073 & 0.71073 & 0.71073 & 0.71073 \\
\hline Crystal system & Monoclinic & Monoclinic & Monoclinic & Monoclinic & Monoclinic & Monoclinic \\
\hline Space group & $\mathrm{P} 2{ }_{1} / \mathrm{c}$ & $\mathrm{P} 2_{1} / \mathrm{c}$ & $\mathrm{P} 2_{1} / \mathrm{n}$ & $\mathrm{P} 2_{1} / \mathrm{c}$ & $\mathrm{P} 2_{1} / \mathrm{c}$ & $\mathrm{P} 2_{1} / \mathrm{c}$ \\
\hline $\mathrm{a}(\AA)$ & $9.1341(8)$ & $10.1638(6)$ & $8.9107(8)$ & $16.6693(12)$ & $10.6823(5)$ & $10.336(2)$ \\
\hline $\mathrm{b}(\AA)$ & $16.4268(15)$ & $19.5791(11)$ & $16.0155(14)$ & $10.4115(7)$ & $18.6756(8)$ & $19.755(4)$ \\
\hline$c(\AA)$ & $18.2838(16)$ & $20.5215(11)$ & $14.5532(13)$ & $12.4751(9)$ & $14.7019(7)$ & $20.644(4)$ \\
\hline$\alpha(\operatorname{deg})$ & 90.00 & 90.00 & 90.00 & 90.00 & 90.00 & 90.00 \\
\hline$\beta(\operatorname{deg})$ & $103.371(1)$ & $95.071(1)$ & $90.034(1)$ & $106.366(1)$ & $109.092(1)$ & $93.835(4)$ \\
\hline$\gamma(\mathrm{deg})$ & 90.00 & 90.00 & 90.00 & 90.00 & 90.00 & 90.00 \\
\hline $\mathrm{V}\left(\AA^{3}\right)$ & $2,669.0(4)$ & $4067.8(4)$ & $2,076.9$ (3) & $2,077.4(3)$ & $2,771.7(2)$ & $4,205.8(14)$ \\
\hline $\mathrm{Z}$ & 2 & 8 & 4 & 4 & 4 & 4 \\
\hline $\mathrm{D}_{\text {calcd }}\left(\mathrm{Mg} / \mathrm{m}^{3}\right)$ & 1.268 & 1.340 & 1.357 & 1.564 & 1.402 & 1.781 \\
\hline $\begin{array}{l}\text { Absorp coeff } \\
\left(\mathrm{mm}^{-1}\right)\end{array}$ & 0.86 & 1.11 & 1.09 & 3.01 & 3.60 & 6.625 \\
\hline $\mathrm{F}(000)$ & 1,064 & 1704 & 884 & 988 & 1192 & 2208 \\
\hline Crystal size (mm) & $0.28 \times 0.13 \times 0.09$ & $0.25 \times 0.21 \times 0.09$ & $0.28 \times 0.17 \times 0.10$ & $0.21 \times 0.12 \times 0.07$ & $0.27 \times 0.13 \times 0.10$ & $0.25 \times 0.16 \times 0.11$ \\
\hline$\theta$ Range (deg) & $2.3-26.0$ & $2.3-24.9$ & $2.5-24.5$ & $2.6-26.0$ & $2.3-26.0$ & $2.2-24.5$ \\
\hline $\begin{array}{l}\text { No. of reflns } \\
\text { collected }\end{array}$ & 15,743 & 23,659 & 15,347 & 14,498 & 20,676 & 26,672 \\
\hline No. of indep reflns & 5243 & 7,178 & 4,071 & 4,034 & 5,455 & 8,310 \\
\hline $\begin{array}{c}\text { No. of data/ } \\
\text { restraints/params }\end{array}$ & $\begin{array}{l}5,243 / 7 / 313 \\
\left(R_{\text {int }}=0.026\right)\end{array}$ & $\begin{array}{l}7,178 / 0 / 443 \\
\left(R_{\text {int }}=0.042\right)\end{array}$ & $\begin{array}{l}4,071 / 0 / 232 \\
\left(R_{\text {int }}=0.038\right)\end{array}$ & $\begin{array}{l}4,034 / 0 / 231 \\
\left(R_{\text {int }}=0.023\right)\end{array}$ & $\begin{array}{l}5,455 / 0 / 277 \\
\left(R_{\text {int }}=0.024\right)\end{array}$ & $\begin{array}{l}8,310 / 0 / 441 \\
\left(R_{\text {int }}=0.055\right)\end{array}$ \\
\hline GOF on $\mathrm{F}^{2}$ & 1.05 & 0.98 & 1.01 & 1.04 & 1.04 & 0.97 \\
\hline $\mathrm{R}_{1}(1>2 \operatorname{sigma}(1))$ & 0.045 & 0.038 & 0.036 & 0.030 & 0.025 & 0.039 \\
\hline $\mathrm{wR}_{2}$ & 0.145 & 0.090 & 0.090 & 0.083 & 0.068 & 0.083 \\
\hline
\end{tabular}


Table 2. Selected bond distances $(\AA)$ and angles $\left(^{\circ}\right)$ of cobalt complexes 3a, $\mathbf{4 a}, \mathbf{5 a}$ and $\mathbf{7 a}$.

\begin{tabular}{|c|c|c|c|c|c|c|c|}
\hline $3 \mathbf{a}$ & & $4 a$ & & $5 \mathbf{a}$ & & $7 a$ & \\
\hline \multicolumn{8}{|c|}{ Bond lengths } \\
\hline Co1-N1 & $2.099(2)$ & Co1-N1 & $2.181(2)$ & Co1-N1 & $2.0439(18)$ & Co1-N1 & $2.0667(18)$ \\
\hline Co1-N2 & $2.128(2)$ & Co1-N2 & $2.089(2)$ & Co1-N2 & 2.0498 (18) & Co1-N2 & $2.0514(17)$ \\
\hline Co1-Cl1 & $2.2830(9)$ & Co1-Cl1 & $2.2769(8)$ & Co1-Cl1 & $2.2071(7)$ & Co1-Cl1 & $2.2079(7)$ \\
\hline $\mathrm{Co} 1-\mathrm{Cl} 2$ & $2.3607(8)$ & $\mathrm{Co} 1-\mathrm{Cl} 2$ & $2.4428(8)$ & $\mathrm{Co} 1-\mathrm{Cl} 2$ & $2.2148(7)$ & $\mathrm{Co} 1-\mathrm{Cl} 2$ & $2.2235(7)$ \\
\hline $\mathrm{Co} 1-\mathrm{Cl} 2 \mathrm{~A}$ & $2.4372(8)$ & $\mathrm{Co} 2-\mathrm{N} 4$ & $2.097(2)$ & N2-C7 & $1.290(3)$ & N2-C8 & $1.451(3)$ \\
\hline $\mathrm{Co} 1 \mathrm{~A}-\mathrm{Cl} 2$ & $2.4371(8)$ & $\mathrm{Co} 2-\mathrm{N} 3$ & $2.182(2)$ & N2-C9 & $1.448(3)$ & N2-C6 & 1.285 (3) \\
\hline C5-C6 & $1.493(4)$ & $\mathrm{Co} 2-\mathrm{Cl} 3$ & 2.2709 (8) & C5-C7 & $1.492(3)$ & C5-C6 & $1.492(3)$ \\
\hline N2-C6 & $1.301(4)$ & $\mathrm{Co} 2-\mathrm{Cl} 4$ & $2.4433(8)$ & C7-C8 & $1.490(3)$ & C6-C7 & 1.489 (3) \\
\hline N2-C13 & $1.454(4)$ & N2-C8 & $1.442(3)$ & $\mathrm{C} 1-\mathrm{C} 6$ & $1.491(3)$ & $\mathrm{Br} 1-\mathrm{C} 1$ & $1.888(3)$ \\
\hline \multirow[t]{3}{*}{$\mathrm{C} 6-\mathrm{C} 7$} & $1.489(4)$ & N2-C6 & $1.271(3)$ & & & & \\
\hline & & N4-C27 & $1.438(3)$ & & & & \\
\hline & & $\mathrm{N} 4-\mathrm{C} 25$ & $1.272(3)$ & & & & \\
\hline \multicolumn{8}{|c|}{ Bond angles } \\
\hline N1-Co1-N2 & $76.36(9)$ & N1-Co1-N2 & $77.69(8)$ & N1-Co1-N2 & $80.68(7)$ & N2-Co1-N1 & $80.14(7)$ \\
\hline N1-Co1-Cl1 & $92.08(7)$ & N1-Co1-Cl1 & $91.41(6)$ & N1-Co1-Cl1 & $118.90(6)$ & N1-Co1-Cl1 & $118.64(6)$ \\
\hline N2-Co1-Cl1 & $117.17(7)$ & N2-Co1-Cl1 & $111.97(6)$ & N2-Co1-Cl1 & $115.04(6)$ & N2-Co1-Cl1 & 111.66 (5) \\
\hline N1-Co1-Cl2 & $164.04(8)$ & N1-Co1-Cl2 & $171.60(6)$ & N1-Co1-Cl2 & $107.53(5)$ & N2-Co1-Cl2 & $117.93(5)$ \\
\hline N2-Co1-Cl2 & $98.55(7)$ & N2-Co1-Cl2 & $96.91(6)$ & N2-Co1-Cl2 & $114.54(6)$ & N1-Co1-Cl2 & 110.67 (5) \\
\hline Cl1-Co1-Cl2 & $103.60(3)$ & Cl1-Co1-Cl2 & $96.61(3)$ & Cl1-Co1-Cl2 & $115.44(3)$ & Cl1-Co1-Cl2 & $113.88(3)$ \\
\hline $\mathrm{N} 1-\mathrm{Co} 1-\mathrm{Cl} 2 \mathrm{~A}$ & $85.08(7)$ & $\mathrm{N} 4-\mathrm{Co} 2-\mathrm{N} 3$ & $77.66(8)$ & C7-N2-C9 & 120.43 (19) & N1-C1-Br1 & 116.75 (17) \\
\hline N2-Co1-Cl2A & $125.21(7)$ & $\mathrm{N} 4-\mathrm{Co} 2-\mathrm{Cl} 3$ & $114.12(6)$ & N1-C5-C7 & 115.52 (19) & C2-C1-Br1 & $119.39(19)$ \\
\hline $\mathrm{Cl} 1-\mathrm{Co} 1-\mathrm{Cl} 2 \mathrm{~A}$ & 114.55 (3) & N3-Co2-Cl3 & $94.45(6)$ & C4-C5-C7 & $122.7(2)$ & C7-C6-C5 & 118.39 (19) \\
\hline $\mathrm{Cl} 2-\mathrm{Co} 1-\mathrm{Cl} 2 \mathrm{~A}$ & $85.73(3)$ & $\mathrm{N} 4-\mathrm{Co} 2-\mathrm{Cl} 4$ & $96.58(6)$ & C8-C7-C5 & $118.6(2)$ & C6-N2-C8 & $120.24(18)$ \\
\hline N2-C6-C7 & $125.8(3)$ & N3-Co2-Cl4 & $169.32(6)$ & N2-C7-C8 & $124.9(2)$ & N2-C6-C7 & $125.0(2)$ \\
\hline C6-N2-C13 & $118.9(2)$ & $\mathrm{Cl} 3-\mathrm{Co} 2-\mathrm{Cl} 4$ & $96.12(3)$ & N1-C1-C6 & 116.7 (2) & N2-C6-C5 & $116.58(19)$ \\
\hline N2-C6-C5 & 115.7 (3) & N2-C6-C5 & $120.4(2)$ & N2-C7-C5 & $116.4(2)$ & & \\
\hline
\end{tabular}

Table 3. Selected bond distances $(\AA)$ and angles $\left(^{\circ}\right)$ of nickel complexes $\mathbf{5 b}$ and $\mathbf{6 b}$.

\begin{tabular}{|c|c|c|c|c|c|}
\hline $5 b$ & & $6 b$ & & & \\
\hline \multicolumn{6}{|c|}{ Bond lengths } \\
\hline Ni1-N2 & $2.0383(16)$ & Ni1-N2 & $2.049(4)$ & Br1-Ni1A & $2.4770(8)$ \\
\hline Ni1-N1 & 2.0677 (17) & Ni1-N1 & $2.092(3)$ & Br3-C1 & $1.887(5)$ \\
\hline Ni1-O1 & $2.1478(15)$ & Ni1-Br2 & $2.4153(8)$ & Br5-Ni2A & $2.5076(8)$ \\
\hline Ni1-Br1 & $2.4376(3)$ & Ni1-Br1A & $2.4770(8)$ & Br6-C19 & $1.875(5)$ \\
\hline Ni1-Br2 & $2.4602(3)$ & Ni1-Br1 & $2.5230(8)$ & & \\
\hline C5-C7 & $1.492(3)$ & Ni2-N4 & $2.038(3)$ & & \\
\hline C7-C8 & $1.494(3)$ & Ni2-N3 & $2.080(3)$ & & \\
\hline N2-C7 & $1.286(3)$ & Ni2-Br4 & $2.4214(9)$ & & \\
\hline N2-C9 & $1.445(2)$ & Ni2-Br5 & $2.4777(8)$ & & \\
\hline $\mathrm{C} 1-\mathrm{C} 6$ & $1.489(3)$ & Ni2-Br5A & $2.5075(8)$ & & \\
\hline \multicolumn{6}{|c|}{ Bond angles } \\
\hline N2-Ni1-N1 & $80.22(6)$ & N2-Ni1-N1 & $80.27(13)$ & N4-Ni2-Br4 & $107.02(10)$ \\
\hline N2-Ni1-Br1 & $106.94(5)$ & N2-Ni1-Br2 & $107.62(9)$ & N3-Ni2-Br4 & $90.38(10)$ \\
\hline N1-Ni1-Br1 & $91.16(5)$ & N1-Ni1-Br2 & $90.87(9)$ & N4-Ni2-Br5 & $104.32(10)$ \\
\hline N2-Ni1-Br2 & $111.61(5)$ & N2-Ni1-Br1A & $104.84(10)$ & N3-Ni2-Br5 & $90.36(10)$ \\
\hline N1-Ni1-Br2 & $90.48(5)$ & N1-Ni1-Br1A & $90.92(9)$ & Br4-Ni2-Br5 & $148.29(3)$ \\
\hline Br1-Ni1-Br2 & $141.124(13)$ & Br2-Ni1-Br1A & $147.32(3)$ & N4-Ni2-Br5A & $100.41(9)$ \\
\hline C5-C7-C8 & $118.23(17)$ & N2-Ni1-Br1 & $100.45(9)$ & N3-Ni2-Br5A & $176.73(10)$ \\
\hline $\mathrm{N} 2-\mathrm{C} 7-\mathrm{C} 8$ & 125.39 (19) & N1-Ni1-Br1 & $176.28(9)$ & Br4-Ni2-Br5A & $92.55(2)$ \\
\hline N1-C1-C6 & 118.41 (19) & Br2-Ni1-Br1 & $92.38(2)$ & Br5-Ni2-Br5A & $86.39(2)$ \\
\hline C2-C1-C6 & $120.9(2)$ & Br1A-Ni1-Br1 & $85.37(2)$ & N2-C6-C5 & $120.1(4)$ \\
\hline C7-N2-C9 & 119.55 (16) & N4-Ni2-N3 & 80.09 (13) & N1-C1-Br3 & $117.2(3)$ \\
\hline
\end{tabular}

Cobalt complexes $4 \mathbf{a}, \mathbf{5 a}$, and $7 \mathbf{a}$ with a substituent $\left(4 \mathbf{a}: \mathrm{CH}_{3} ; \mathbf{5 a}: \mathrm{CH}_{3} ; \mathbf{7 a}\right.$ : $\left.\mathrm{Br}\right)$ at the 6-position of the pyridine ring produced the solid structures with a single ligand coordinated to the central metal. The asymmetric unit of $4 \mathbf{a}$ contains two independent, closely resembling molecules (Figure 1), whereas only one molecule is found in the asymmetric unit of complexes $5 \mathbf{a}$ (Figure 2) and 7a (Figure 3). In complex $4 \mathbf{a}$ crystallizing in a monoclinic space group $\mathrm{P} 2{ }_{1} / \mathrm{c}$, the ligand system adopts a cisoid 
conformation that allows both the nitrogen centers N1 and N2 (N3 and N4) to coordinate to the cobalt atom. The metal-nitrogen bond lengths are $2.181 \AA$ (Co1-N1) and $2.089 \AA$ (Co1-N2), and the $\mathrm{Co}-\mathrm{Cl}$ bond lengths are $2.2769 \AA$ (Co1-Cl1) and $2.4428 \AA$ (Co1-Cl2). In complex $4 a$, the cobalt atom has distorted tetrahedral coordination geometry with $\mathrm{N} 1-\mathrm{Co} 1-\mathrm{N} 2$ angle of $77.69^{\circ}$ and $\mathrm{Cl1}-\mathrm{Co} 1-\mathrm{Cl} 2$ angle of $96.61^{\circ}$. The 2,6-diisopropylphenyl substituent at the imine nitrogen atom is almost perpendicular to the plane of the pyridine moiety (angle between the planes: $89.84^{\circ}$ ). The angle of N2-C5-C6 is $120.4^{\circ}$ when there is a $\mathrm{H}$ atom in the imino bridge. Crystallizing in a monoclinic space group $\mathrm{P} 2_{1} / \mathrm{n}$ and $\mathrm{P} 2_{1} / \mathrm{c}$, complexes $5 \mathbf{a}$ and $\mathbf{7 a}$ with $\mathrm{CH}_{3}$ group in the imino bridge have a smaller $\mathrm{N} 2-\mathrm{C} 7-\mathrm{C} 5$ angle of $116.4^{\circ}$ and N2-C6-C5 angle of $116.58^{\circ}$ than those in complex 4a. Meanwhile, they have longer N-Co bond distances (5a: $2.0439 \AA$, $2.0498 \AA$; 7a: $2.0667 \AA, 2.0514 \AA$ ), shorter $\mathrm{Co}-\mathrm{Cl}$ bond distances (5a: $2.2071 \AA, 2.2148 \AA ; 7 \mathbf{a}: 2.2079 \AA, 2.2235 \AA$ ), and bigger angles of N1-Co1-N2 (5a, 80.68 ; 7a, $80.14^{\circ}$ ) and Cl1-Co1-Cl2 (5a, 115.44; 7a, 113.88) than complex 4a. The 2,6-diisopropylphenyl substituent at the imine nitrogen atom is also perpendicular to the plane of the pyridine moiety (angle between the planes: $5 \mathbf{a}, 89.56^{\circ} ; 7 \mathrm{a}, 83.97^{\circ}$ ). However, ligand $\mathbf{L} 2$ and $\mathbf{L} 3$ ( $\mathrm{H}$ atom at 6-position of the pyridine ring) results in the formation of a chloro-bridged dimer. In complex $\mathbf{2 a}$, reported in a previous report [22], the $\mathrm{Co}-\mathrm{N}$ bond distances are 2.093 and $2.123 \AA$, which are longer than those in $\mathbf{5 a}$ and $7 \mathbf{a}$ but shorter than that in complex $4 \mathbf{a}$; however, the $\mathrm{Co}-\mathrm{Cl}$ bond distance exhibits the converse regularity $(4 \mathbf{a}>\mathbf{2 a}>\mathbf{5 a} \approx 7 \mathbf{a})$. In complex 3a (Figure 4), with phenyl group in the imino bridge, the coordination geometry of the central metal atom can be best described as distorted trigonal-bipyramidal with two equivalent half molecules in an overall $\mathrm{C}_{2}$-symmetric framework. The pyridyl nitrogen atom (N1) and $\mathrm{Cl}$ mark the apical centers of the distorted trigonal bipyramid. The pyridine nitrogen (N1) and the other constituent of the chloro-bridge ( $\mathrm{Cl} 2)$ occupy the axial coordination sites. Complex 3a has a bipyramidal structure most distorted towards a square-based pyramid, with the most acute angle $\left(164.04^{\circ}\right)$ in the vertical axis (N1-Co1-Cl2) and one of the equatorial angles extended to $125.21^{\circ}$.

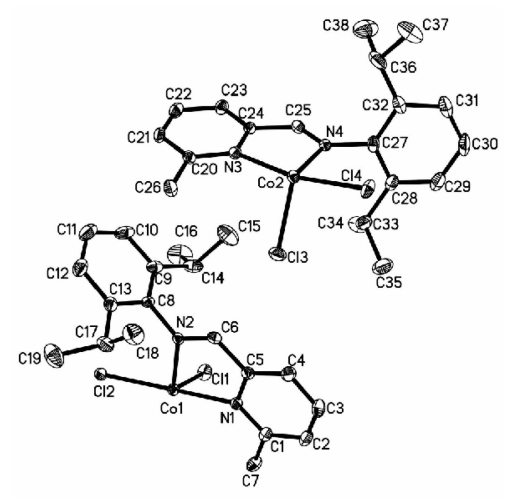

Figure 1. ORTEP view of complex $4 \mathbf{a}$, drawn at $35 \%$ of probability. Hydrogen atoms were omitted for clarity.

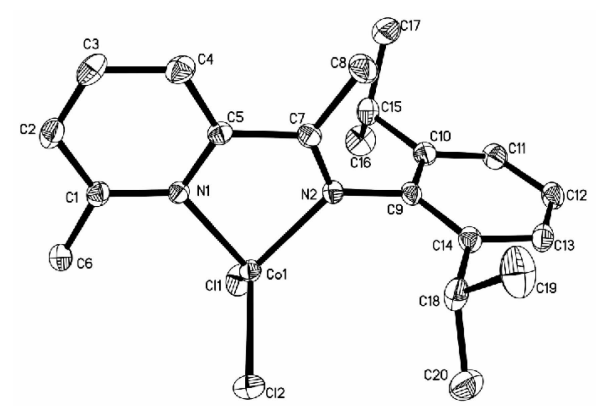

Figure 2. ORTEP view of complex $5 \mathbf{a}$, drawn at $35 \%$ of probability. Hydrogen atoms were omitted for clarity. 


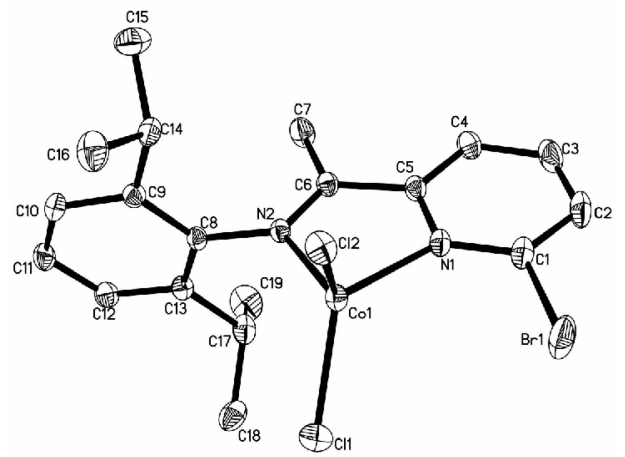

Figure 3. ORTEP view of complex 7a, drawn at 35\% of probability. Hydrogen atoms were omitted for clarity.

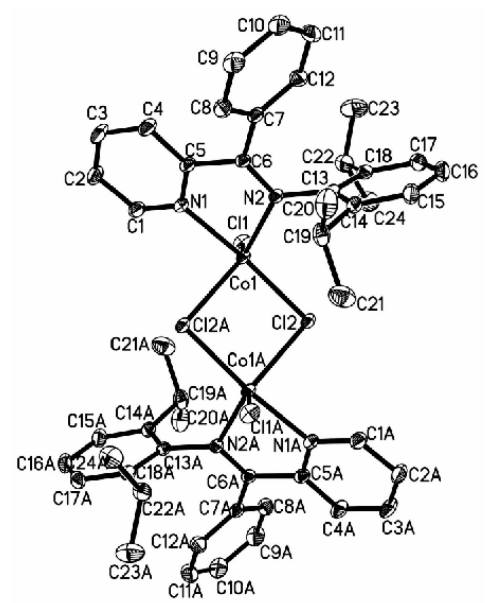

Figure 4. ORTEP view of complex 3a, drawn at 35\% of probability. Hydrogen atoms and one $\mathbf{E t}_{2} \mathbf{O}$ molecule were omitted for clarity.

Nickel complex $5 \mathbf{b}$ crystallized from THF in a monoclinic space group $\mathrm{P} 2_{1} / c$, producing the solid structures with a single ligand and one THF molecule coordinated to the metal center. In complex $\mathbf{5 b}$ (Figure 5), the nickel is five coordinate, and the coordination sphere could be best described as a distorted trigonal bypyramidal with the equatorial plane occupied by the imino $\mathrm{N}$ atom and the two bromine atoms. The axial positions are coordinated by the pyridine $\mathrm{N}$ atom and by the $\mathrm{O}$ atom of the THF molecule. A Br-Ni-Br angle of $141.13^{\circ}$ was observed within the equatorial plane and a "chelating" angle of $\mathrm{N} 1-\mathrm{Ni}-\mathrm{N} 2$ is $80.22^{\circ}$. The distance of $\mathrm{Ni}-\mathrm{O}$ bond is $2.148 \AA$, which is a little longer than those of the N-Ni bond (2.0383, $2.0677 \AA$ ), indicating a very strongly bound THF ligand. Complex $\mathbf{6 b}$ (Figure 6) with $\mathrm{Br}$ at the 6-position of the pyridine ring crystallizes as centrosymmetric dimmers with two ligand nitrogen atoms, one terminal bromine and two bridging bromine atoms forming the coordination sphere around the five-coordinate nickel center. Complex $\mathbf{6 b}$ contains the halves of two independent and nearly identical molecules, which is similar to complex $4 \mathrm{~b}$ reported in the literature in that both have nearly the same Co-N bond distance (4b: 2.086, $2.042 \AA ; 6 \mathbf{b}: 2.092,2.049 \AA$ ), Ni-Br bond distance (4b: 2.5411, $2.4136 \AA$; 6 b: $2.4214,2.4777 \AA$ ), and N-Ni-N angle (4b: $\left.80.29^{\circ} ; 6 \mathbf{b}: 80.27^{\circ}\right)$. 


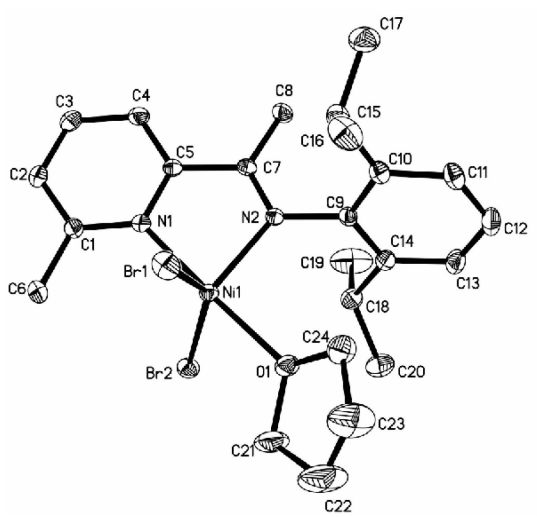

Figure 5. ORTEP view of complex $5 \mathbf{b}$, drawn at $35 \%$ of probability. Hydrogen atoms and one THF molecule were omitted for clarity.

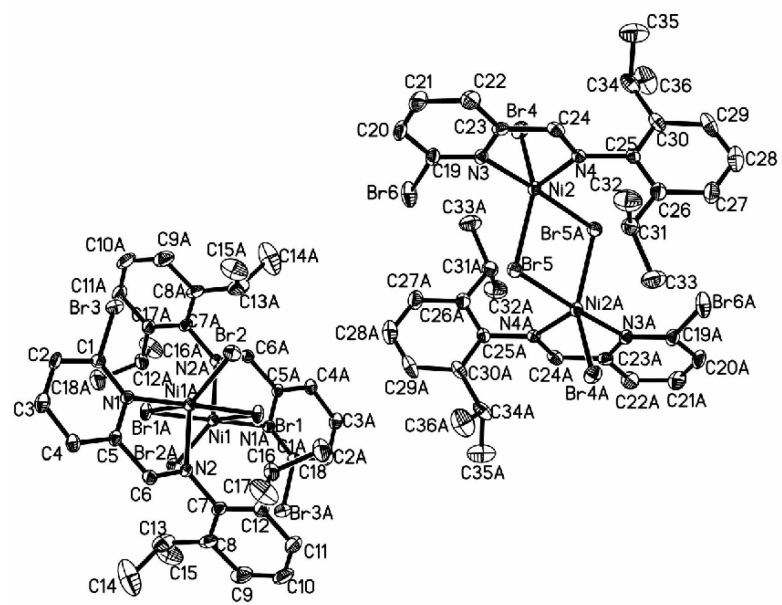

Figure 6. ORTEP view of complex $\mathbf{6 b}$, drawn at $35 \%$ of probability. Hydrogen atoms were omitted for clarity.

\subsection{Solution Polymerization of 1,3-Butadiene}

Cobalt complexes could polymerize 1,3-butadiene activated by ethylaluminum sesquichloride (EASC). Herein, we mainly discuss the influences of the $\mathrm{R}_{1}$ group $\left(\mathrm{H}, \mathrm{CH}_{3}\right.$ and $\mathrm{Br}$ ) at the 6-position of the pyridine ring and $\mathrm{R}_{2}$ group $\left(\mathrm{H}\right.$, aldimine; $\mathrm{CH}_{3}$, ketimine) in the imino bridge on catalytic activity and selectivity of the complexes. The polymerization results are summarized in Table 4.

Table 4. Polymerization of 1,3-butadiene with Co (II) complex/EASC catalyst ${ }^{\text {a }}$.

\begin{tabular}{|c|c|c|c|c|c|c|c|}
\hline \multirow{2}{*}{ Run } & \multirow{2}{*}{ Complex } & \multirow{2}{*}{ Yield (\%) } & \multirow{2}{*}{$M_{\mathbf{n}}{ }^{\mathbf{b}} \times 10^{-4}$} & \multirow{2}{*}{$M_{\mathrm{w}} / M_{\mathrm{n}}{ }^{b}$} & \multicolumn{3}{|c|}{ Microstructure $^{\mathrm{c}}(\%)$} \\
\hline & & & & & Cis-1,4 & 1.2 & Trans-1,4 \\
\hline 1 & $1 a$ & 56.9 & 23.0 & 2.4 & 98.1 & 1.0 & 0.9 \\
\hline 2 & $2 a$ & 51.6 & 27.7 & 2.1 & 98.2 & 1.0 & 0.8 \\
\hline 3 & $3 \mathbf{a}$ & 92.3 & 9.0 & 3.3 & 96.5 & 1.6 & 1.9 \\
\hline 4 & $4 a$ & 42.9 & 31.5 & 1.9 & 98.3 & 0.9 & 0.8 \\
\hline 5 & $5 \mathbf{a}$ & 31.9 & 33.8 & 1.7 & 98.5 & 0.9 & 0.6 \\
\hline 6 & $6 a$ & 92.8 & 10.9 & 3.0 & 97.2 & 1.4 & 1.4 \\
\hline 7 & $7 a$ & 90.8 & 12.0 & 3.2 & 97.0 & 1.4 & 1.6 \\
\hline
\end{tabular}

a Polymerization in hexane at $20^{\circ} \mathrm{C}$ for $15 \mathrm{~min},[\mathrm{Bd}]=1.85 \mathrm{~mol} / \mathrm{L},[\mathrm{BD}] /[\mathrm{Co}]=1000,[\mathrm{Al}] /[\mathrm{Co}]=50$.

${ }^{\mathrm{b}}$ Determined by GPC (THF, PSt calibration). ${ }^{\mathrm{c}}$ The microstructure was determined by FTIR. 
The substituent $\left(\mathrm{R}_{1}\right)$ at the 6-position of the pyridine ring significantly influence the catalytic performances of the complexes. For the aldimine- and ketimine-based cobalt complexes, the introduction of halogen atom $(\mathrm{Br})$ at 6-position of the pyridine ring afforded polymers in higher yields but with relatively lower molecular weight than the complexes without substituent. The complexes with $\mathrm{CH}_{3}$ group produced polymers in lower yield but with higher molecular weight and narrower molecular weight distribution. For instance, the polymerization of 1,3-butadiene with aldimine cobalt complex $\mathbf{6 a}\left(\mathrm{R}_{1}=\mathrm{Br}\right)$ produced polymer in $92.8 \%$ yield, which is much higher than complexes 1a $\left(\mathrm{R}_{1}=\mathrm{H}, 56.9 \%\right)$ and $\mathbf{4 a}\left(\mathrm{R}_{1}=\mathrm{CH}_{3}, 42.9 \%\right)$. Similar to aldimine cobalt complexes, ketimine cobalt complex $7 \mathrm{a}\left(\mathrm{R}_{1}=\mathrm{Br}\right)$ displayed higher catalyst activity (polymer yield: $\left.90.8 \%\right)$ than $2 \mathrm{a}\left(\mathrm{R}_{1}=\mathrm{H}\right.$, $51.6 \%)$ and $5 \mathrm{a}\left(\mathrm{R}_{1}=\mathrm{CH}_{3}, 31.9 \%\right)$. On the other hand, the molecular weights of polybutadienes obtained by these cobalt complexes exhibited the converse regularity (in the orders of $\mathbf{6 a}<\mathbf{1 a}<\mathbf{4 a}$ and $7 \mathbf{a}<2 \mathbf{a}<5 \mathbf{a}$ ). These results could be explained by the idea that the electron-withdrawing group $(\mathrm{Br})$ at the 6-position reduces the electron density of central metal, and the increased Lewis acidic character facilitates the coordination of 1,3-butadiene molecule, leading to the increment of chain propagation rate. Moreover, due to the electron-contributing and steric hindrance of the $\mathrm{CH}_{3}$ group, the coordination reaction between 1,3-butadiene and the cobalt center was retarded [10] . The $\mathrm{CH}_{3}$ group can serve to raise polymer molecular weight $\left(4 \mathbf{a}, 31.5 \times 10^{4} ; \mathbf{5 a}, 33.8 \times 10^{4}\right)$ and regulate molecular weight distribution $(4 \mathbf{a}, 1.9 ; 5 \mathbf{a}, 1.7)$, suggesting that electron-contributing substituent at 6-position of the pyridine ring retard chain transfer reaction to some extent.

The substituent $\left(R_{2}\right)$ in the imino bridge of the iminopyridine cobalt(II) complex was also investigated. Complex 3a with a phenyl group exhibited much higher activity than $1 a\left(R_{2}=H\right)$ and 2a $\left(\mathrm{R}_{2}=\mathrm{CH}_{3}\right)$. This result indicates that the conjugation of phenyl and imine groups can stabilize the active centers and cause the increased chain propagation rate. In olefin catalysts based on bisiminopyridine transition metal complexes, replacing a ketimine $\left(\mathrm{R}_{2}=\mathrm{CH}_{3}\right)$ with an aldimine $\left(\mathrm{R}_{1}=\mathrm{H}\right)$ usually leads to difference in molecular weight and molecular weight distribution, particularly the productivity, and usually the catalysts based on an ketimine ligand are approximately one order of magnitude more active than those based on an aldimine ligand [25]. In 1,3-butadiene polymerization, bisiminopyridine cobalt complexes based on ketimine ligand also showed higher catalytic activity than their aldimine counterparts [9]. In the present study, however, iminopyridine cobalt(II) complexes based on aldimine produced the polybutadiene in a higher polymer yield, smaller molecular weight and broader molecular weight distribution than the ketimine complexes, and the results were as follows: $1 \mathrm{a}\left(\mathrm{R}_{1}=\mathrm{H}, 56.9 \%\right)>\mathbf{2 a}\left(\mathrm{R}_{1}=\mathrm{H}, 51.6 \%\right), \mathbf{4 a}\left(\mathrm{R}_{1}=\mathrm{CH}_{3}, 42.9 \%\right)>5 \mathbf{a}\left(\mathrm{R}_{1}=\mathrm{CH}_{3}, 31.9 \%\right)$ and $6 \mathbf{a}\left(\mathrm{R}_{1}=\mathrm{Br}, 92.8 \%\right)>7 \mathrm{a}\left(\mathrm{R}_{1}=\mathrm{Br}, 90.8 \%\right)$. This is due to the fact that, during the activation with EASC, the $-\mathrm{CH}=\mathrm{N}$ groups of the complexes can be deprotonated to yield an anionic amide ligand. This anionic amide ligand is in the form of an ion pair or in the free form, resulting in higher polymerization activity $[12,26]$.

The polybutadienes obtained by these iminopyridine-based cobalt(II) complexes had high cis-1,4 content greater than $96 \%$. Complexes $4 \mathbf{a}$ and $5 \mathbf{a}$ with $\mathrm{CH}_{3}$ at 6-position of the pyridine ring produced polymers with higher cis-1,4 content (4a, 98.3\%; $\mathbf{5 a}, 98.5 \%)$ than the substituent-free (1a, $98.1 \%$; 2a, 98.2\%; 3a, 96.5\%) and Br-containing complexes (6a, 97.2\%; 7a, 97.0\%). However, $\mathrm{R}_{2}$ groups in the imino of iminopyridine cobalt(II) complexes did not influence selectivity significantly.

The nature of the metal center had a large influence on the catalytic performance. In general, cobalt-based catalysts were more active than the corresponding nickel-based analogs; under the same conditions, except for a prolonged polymerization time by $3 \mathrm{~h}$ employed here, iminopyridine-based nickel complexes produced polymers in $29.2 \%$ to $79.3 \%$ yields. All the nickel complexes $\mathbf{1 b} \mathbf{b}-\mathbf{7 b}$ yielded polymers with much lower molecular weights $(7000-9900 \mathrm{~g} / \mathrm{mol})$, narrower molecular weight distributions (1.72-2.67), and lower cis-1,4 contents (79.3\%-90.7\%) than their cobalt counterparts (Entry 8-14 in Table 4). Similarly, as for the nickel complexes, the aldimine $\left(R_{2}=H\right)$ complexes showed higher catalyst activity than the corresponding ketimine $\left(\mathrm{R}_{2}=\mathrm{CH}_{3}\right)$ complexes in the orders: $\mathbf{1 b}$ $\left(\mathrm{R}_{1}=\mathrm{H}, 69.7 \%\right.$ conv. $)>\mathbf{2} \mathbf{b}\left(\mathrm{R}_{1}=\mathrm{H}, 35.8 \%\right.$ conv. $), \mathbf{4 b}\left(\mathrm{R}_{1}=\mathrm{CH}_{3}, 75.4 \%\right.$ conv. $)>5 \mathbf{b}\left(\mathrm{R}_{1}=\mathrm{CH}_{3}, 47.1 \%\right.$ 
conv.) and $\mathbf{6 b}\left(\mathrm{R}_{1}=\mathrm{Br}, 79.3 \%\right.$ conv. $)>7 \mathbf{b}\left(\mathrm{R}_{1}=\mathrm{Br}, 72.8 \%\right.$ conv. $)$. Moreover, nickel complexes $(\mathbf{6 b}, \mathbf{7 b})$ with $\mathrm{Br}$ at 6-position of pyridine ring exhibited a higher catalyst activity than the cobalt complexes described. However, $\mathbf{3 b}$ with phenyl in the imino bridge showed the lowest catalyst activity, quite different from $3 a$ with the same ligand, indicating that phenyl group in the complex could retard the chain propagation and chain transfer reaction to produce the polymer with lower yield, higher molecular weight, and narrower molecular weight distribution (Entry 10 in Table 5). The results in Table 5 demonstrated that the ligand environment did not significantly influence molecular weight and molecular weight distribution of the obtained polymers, but remarkably influenced the stereoregularity of the polybutadienes. The aldimine complexes produced polymers with lower cis-1,4 content, more trans-1,4 content and nearly invariable 1,2 content compared with ketimine complexes. The ${ }^{13} \mathrm{C}$ NMR spectra of the polymers obtained with $\mathbf{6 a}$ and $\mathbf{6 b}$ are shown in Figure 7 . It is clear that the nickel complex produced the polymers with higher trans-1,4 content than the cobalt complex sharing the same corresponding ligand L6.

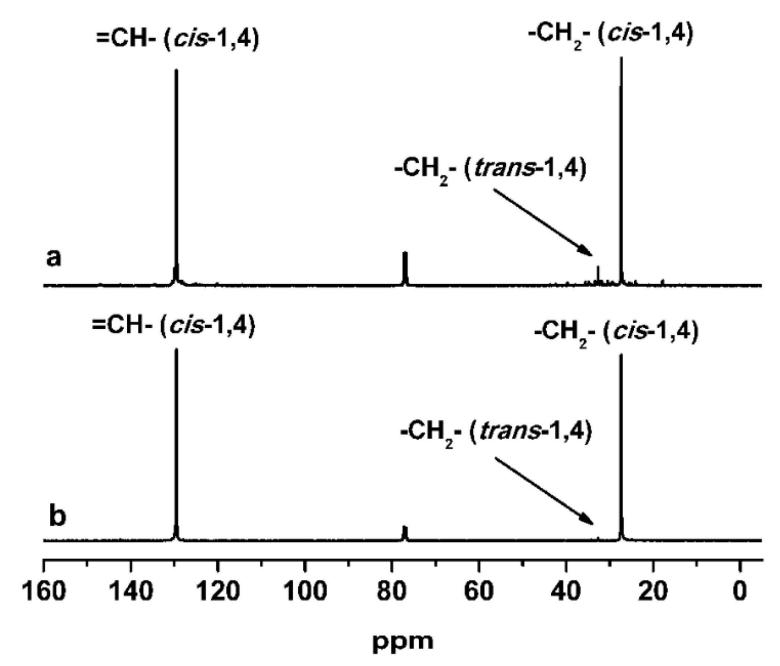

Figure $7 .{ }^{13} \mathrm{C}$ NMR spectra of polybutadienes obtained by $6 \mathrm{a} / \mathrm{EASC}(\mathbf{a})$ and $6 \mathrm{~b} / \mathrm{EASC}(\mathbf{b})$.

Table 5. Polymerization of 1,3-butadiene with Ni (II) complexes ${ }^{\text {a }}$.

\begin{tabular}{cccccccc}
\hline \multirow{2}{*}{ Run } & \multirow{2}{*}{ Complex } & \multirow{2}{*}{ Yield (\%) } & \multirow{2}{*}{$\boldsymbol{M}_{\mathbf{n}} \mathbf{b}$} & \multirow{2}{*}{$\boldsymbol{M}_{\mathbf{w}} / \boldsymbol{M}_{\mathbf{n}} \mathbf{b}$} & \multicolumn{3}{c}{ Microstructure $^{\mathbf{c}} \mathbf{( \% )}$} \\
\cline { 6 - 7 } & & & & & Cis-1,4 & $\mathbf{1 . 2}$ & Trans-1,4 $^{-1}$ \\
\hline 8 & $\mathbf{1 b}$ & 69.7 & 8,100 & 2.40 & 87.1 & 3.4 & 9.5 \\
9 & $\mathbf{2 b}$ & 35.8 & 8,700 & 1.87 & 91.6 & 3.5 & 4.9 \\
10 & $\mathbf{3 b}$ & 29.2 & 9,900 & 1.72 & 90.7 & 3.8 & 5.5 \\
11 & $\mathbf{4 b}$ & 75.4 & 7,100 & 2.38 & 84.1 & 1.7 & 14.2 \\
12 & $\mathbf{5 b}$ & 47.1 & 8,500 & 1.87 & 90.7 & 3.3 & 6.0 \\
13 & $\mathbf{6 b}$ & 79.3 & 7,200 & 2.67 & 79.3 & 1.3 & 19.4 \\
14 & $\mathbf{7 b}$ & 72.8 & 7,000 & 2.17 & 87.1 & 2.3 & 10.6 \\
\hline
\end{tabular}

by GPC (THF, PSt calibration). ${ }^{\mathrm{c}}$ The microstructure was determined by FTIR.

\section{Conclusions}

Structurally well-defined iminopyridine ligated cobalt and nickel complexes were synthesized, and their catalytic behaviors in 1,3-butadiene polymerization were investigated. Activated by EASC, the cobalt complexes exhibited high cis-1,4 selectivity (up to 98.5\%), affording high molecular weight polybutadienes $\left(M_{\mathrm{n}}=9.0-33.8 \times 10^{4}\right)$, while the nickel complexes produced polymers with low molecular weight $\left(M_{n}=7000-9000\right)$ and cis-1,4 stereoregularity $(79.3 \%-91.6 \%)$. In both cases of 
Co- and Ni-based complexes, the aldimine-based complexes showed higher catalyst activity than their ketimine counterparts.

Acknowledgments: The authors appreciate financial support from National Basic Research Program of China, Grant No. 2015CB654700 (2015CB654702), The CAS Hundred Talents Program (Y220011001), the Funds for National Natural Scientific Foundation of China (No. 51203092, 51203147, and 51473156) and Jilin Provincial Research Fund for Basic Research, China (No. 20130102007JC).

Author Contributions: Xiangyu Jia performed the experiments and analyzed the data. Quanquan Dai contributed to the analysis of the results and to the writing of the manuscript. Yanming Hu contributed to the discussion section and revised the paper. Feng Yang, Chenxi Bai and Xuequan Zhang provided the funds.

Conflicts of Interest: The authors declare no conflict of interest.

\section{Appendix}

CCDC numbers 893455(3a), 893456(4a), 893457(5a), 893458(7a), 893459(5b) and 893460(6b) contain the supplementary crystallographic data for this paper. This data can be obtained free of charge from The Cambridge Crystallographic Data Centre via www.ccdc.cam.ac.uk/data_request/cif.

\section{References}

1. Thiele, S.K.H.; Wilson, D.R. Alternate transition metal complex based diene polymerization. J. Macromol. Sci. Polym. Rev. 2003, 43, 581-628. [CrossRef]

2. Cooper, W. Aspects of mechanism of coordination polymerization of conjugated dienes. Ind. Eng. Chem. Prod. Res. Dev. 1970, 9, 457-466. [CrossRef]

3. Furukawa, J. Mechanism of diene polymerization. Pure Appl. Chem. 1975, 42, 495-508. [CrossRef]

4. Oehme, A.; Gebauer, U.; Gehrke, K.; Lechner, M.D. The influence of ageing and polymerization conditions on the polymerization of butadiene using a neodymium catalyst system. Angew. Makromol. Chem. 1996, 235, 121-130. [CrossRef]

5. Gong, D.R.; Dong, W.M.; Hu, Y.M.; Bi, J.F.; Zhang, X.Q.; Jiang, L.S. Syndiotactically enriched 1,2-selective polymerization of 1,3-butadiene initiated by iron catalysts based on a new class of donors. Polymer 2009, 50, 5980-5986. [CrossRef]

6. Lu, J.; Hu, Y.M.; Zhang, X.; Bi, J.F.; Dong, W.M.; Jiang, L.S.; Huang, B.T. Fe(2-EHA) $)_{3} / \mathrm{Al}(\mathrm{i}-\mathrm{Bu})_{3} /$ hydrogen phosphite catalyst for preparing syndiotactic 1,2-polybutadiene. J. Appl. Polym. Sci. 2006, 100, 4265-4269. [CrossRef]

7. Endo, K.; Kitagawa, T.; Nakatani, K. Effect of an alkyl substituted in salen ligands on 1,4-cis selectivity and molecular weight control in the polymerization of 1,3-butadiene with (salen)Co(II). J. Polym. Sci. Polym. Chem. 2006, 44, 4088-4094. [CrossRef]

8. Chandran, D.; Kwak, C.H.; Ha, C.S.; Kim, I. Polymerization of 1,3-butadiene by bis(salicylaldiminate)cobalt(II) catalysts combined with organoaluminium cocatalysts. Catal. Today 2008, 131, 505-512. [CrossRef]

9. Gong, D.R.; Wang, B.L.; Cai, H.G.; Zhang, X.Q.; Jiang, L.S. Synthesis, characterization and butadiene polymerization studies of cobalt(II) complexes bearing bisiminopyridine ligand. J. Organomet. Chem. 2011, 696, 1584-1590. [CrossRef]

10. Cariou, R.; Chirinos, J.J.; Gibson, V.C.; Jacobsen, G.; Tomov, A.K.; Britovsek, G.J.P.; Whitea, A.J.P. The effect of the central donor in bis(benzimidazole)-based cobalt catalysts for the selective cis-1,4-polymerisation of butadiene. Dalton Trans. 2010, 39, 9039-9045. [CrossRef] [PubMed]

11. Gong, D.R.; Jia, X.Y.; Wang, B.L.; Zhang, X.Q.; Jiang, L.S. Synthesis, characterization, and butadiene polymerization of iron(III), iron(II) and cobalt(II) chlorides bearing 2,6-bis(2-benzimidazolyl)pyridyl or 2,6-bis(pyrazol)pyridine ligand. J. Organomet. Chem. 2012, 702, 10-18. [CrossRef]

12. Appukuttan, V.; Zhang, L.; Ha, J.Y.; Chandran, D.; Bahuleyan, B.K.; Ha, C.S.; Kim, I. Stereospecific polymerizations of 1,3-butadiene catalyzed by $\mathrm{Co}$ (II) complexes ligated by 2,6-bis(benzimidazolyl)pyridines. J. Mol. Catal. A Chem. 2010, 325, 84-90. [CrossRef] 
13. Nobbs, J.D.; Tomov, A.K.; Cariou, R.; Gibson, V.C.; White, A.J.P.; Britovsek, G.J.P. Thio-Pybox and Thio-Phebox complexes of chromium, iron, cobalt and nickel and their application in ethylene and butadiene polymerisation catalysis. Dalton Trans. 2012, 41, 5949-5964. [CrossRef] [PubMed]

14. Ai, P.F.; Chen, L.; Guo, Y.T.; Jie, S.Y.; Li, B.G. Polymerization of 1,3-butadiene catalyzed by cobalt(II) and nickel(II) complexes bearing imino- or amino-pyridyl alcohol ligands in combination with ethylaluminum sesquichloride. J. Organomet. Chem. 2012, 705, 51-58. [CrossRef]

15. Jie, S.Y.; Ai, P.F.; Li, B.G. Highly active and stereospecific polymerization of 1,3-butadiene catalyzed by dinuclear cobalt(II) complexes bearing 3-aryliminomethyl-2-hydroxybenzaldehydes. Dalton Trans. 2011, 40, 10975-10982. [CrossRef] [PubMed]

16. Kwag, G.; Jang, Y.C.; Lee, H. Ligand structure and cocatalyst effects on high 1,4-cis polymerization of 1,3-butadiene using Ni-based catalysts. Polym. J. 1999, 31, 1274-1276. [CrossRef]

17. Suzuki, H.; Matsumura, S.; Satoh, Y.; Sogoh, K.; Yasuda, H. Random and block copolymerizations of norbornene with conjugated 1,3-dienes catalyzed by novel Ni compounds involving $\mathrm{N}$ - or O-donated ligands. React. Funct. Polym. 2004, 59, 253-266. [CrossRef]

18. Jang, Y.G.; Choi, D.S.; Han, S. Effects of tris(pentafluorophenyl)borane on the activation of a metal alkyl-free Ni-based catalyst in the polymerization of 1,3-butadiene. J. Polym. Sci. Polym. Chem. 2004, 42, 1164-1173. [CrossRef]

19. Gong, D.R.; Wang, B.L.; Bai, C.X.; Bi, J.F.; Wang, F.; Dong, W.M.; Zhang, X.Q.; Jiang, L.S. Metal dependent control of cis-/trans-1,4 regioselectivity in 1,3-butadiene polymerization catalyzed by transition metal complexes supported by 2,6-bis[1-(iminophenyl)ethyl]pyridine. Polymer 2009, 50, 6259-6264. [CrossRef]

20. Irrgang, T.; Keller, S.; Maisel, H.; Kretschmer, W.; Kempe, R. Sterically demanding iminopyridine ligands. Eur. J. Inorg. Chem. 2007, 2007, 4221-4228. [CrossRef]

21. Laine, T.V.; Piironen, U.; Lappalainen, K.; Klinga, M.; Aitola, E.; Leskela, M. Pyridinylimine-based nickel(II) and palladium(II) complexes: Preparation, structural characterization and use as alkene polymerization catalysts. J. Organomet. Chem. 2000, 606, 112-124. [CrossRef]

22. Bianchini, C.; Giambastiani, G.; Rios, I.G.; Meli, A.; Oberhauser, W.; Sorace, L.; Toti, A. Synthesis of a new polydentate ligand obtained by coupling 2,6-bis(imino)pyridine and (imino)pyridine moieties and its use in ethylene oligomerization in conjunction with iron(II) and cobalt(II) bis-halides. Organometallics 2007, 26, 5066-5078. [CrossRef]

23. Rosa, V.; Carabineiro, S.A.; Aviles, T.; Gomes, P.T.; Welter, R.; Campos, J.M.; Ribeiro, M.R. Synthesis, characterisation and solid state structures of $\alpha$-diimine cobalt(II) complexes: Ethylene polymerisation tests. J. Organomet. Chem. 2008, 693, 769-775. [CrossRef]

24. Song, S.J.; Xiao, T.P.F.; Liang, T.L.; Wang, F.S.; Redshaw, C.; Sun, W.H. Synthesis, characterization and ethylene oligomerization behaviour of 8-(1-aryliminoethylidene)quinaldinylnickel dihalides. Catal. Sci. Technol. 2011, 1, 69-75. [CrossRef]

25. Britovsek, G.J.P.; Bruce, M.; Gibson, V.C.; Kimberley, B.S.; Maddox, P.J.; Mastroianni, S.; McTavish, S.J.; Redshaw, C.; Solan, G.A.; Strömberg, S.; et al. Iron and cobalt ethylene polymerization catalysts bearing 2,6-bis(imino)pyridyl ligands: Synthesis, structures, and polymerization studies. J. Am. Chem. Soc. 1999, 121, 8728-8740. [CrossRef]

26. Zhang, W.; Sun, W.H.; Zhang, S.; Hou, J.; Wedeking, K.; Schultz, S.; Fröhlich, R.; Song, H. Synthesis, characterization, and ethylene oligomerization and polymerization of [2,6-bis(2-benzimidazolyl)pyridyl] chromium chlorides. Organometallics 2006, 25, 1961-1969. [CrossRef]

(C) 2016 by the authors; licensee MDPI, Basel, Switzerland. This article is an open access article distributed under the terms and conditions of the Creative Commons by Attribution (CC-BY) license (http://creativecommons.org/licenses/by/4.0/). 